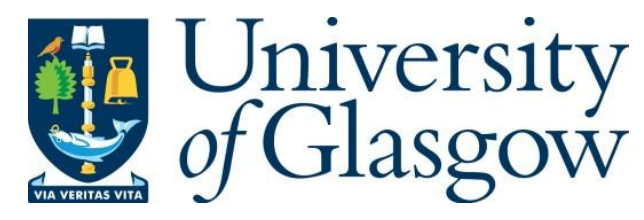

Zare-Behtash, H., Kontis, K., Gongora-Orozco, N., and Takayama, K. (2009) Compressible vortex loops: effect of nozzle geometry. International Journal of Heat and Fluid Flow, 30 (3). pp. 561-576. ISSN 0142-727X (doi:10.1016/j.ijheatfluidflow.2009.02.022)

Copyright @ 2009 Elsevier

A copy can be downloaded for personal non-commercial research or study, without prior permission or charge

Content must not be changed in any way or reproduced in any format or medium without the formal permission of the copyright holder(s)

When referring to this work, full bibliographic details must be given

http://eprints.gla.ac.uk/84650/

Deposited on: 06 January 2014

Enlighten - Research publications by members of the University of Glasgow http://eprints.gla.ac.uk 


\title{
Compressible Vortex Loops: Effect of Nozzle Geometry
}

\author{
H. Zare-Behtash, ${ }^{*}$ K. Kontis, and N. Gongora-Orozco \\ School of MACE, The University of Manchester, M60 1QD, UK \\ K. Takayama \\ Tohoku University, Shock Wave Research Centre, Sendai 980-8577, Japan
}

\begin{abstract}
Vortex loops are fundamental building blocks of supersonic free jets. Isolating them allows for an easier study and better understanding of such flows. The present study looks at the behaviour of compressible vortex loops of different shapes, generated due to the diffraction of a shock wave from a shock tube with different exit nozzle geometries. These include a $15 \mathrm{~mm}$ diameter circular nozzle, two elliptical nozzles with minor to major axis ratios of 0.4 and 0.6 , a $30 \times 30 \mathrm{~mm}$ square nozzle, and finally two exotic nozzles resembling a pair of lips with minor to major axis ratios of 0.2 and 0.5. The experiments were performed for diaphragm pressure ratios of $P_{4} / P_{1}=4,8$, and 12, with $P_{4}$ and $P_{1}$ being the pressures within the high pressure and low pressure compartments of the shock tube, respectively. High-speed schlieren photography as well as PIV measurements of both stream-wise and head-on flows have been conducted.
\end{abstract}

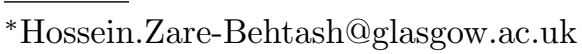




\section{INTRODUCTION}

Non-circular jets provide efficient passive flow control at relatively low cost since they rely solely on changes in the geometry of the nozzle. The applications include improved large and small scale mixing, enhanced combustor performance, noise suppression, heat transfer, and thrust vector control. ${ }^{1}$ Non-circular injectors such as elliptic, triangular, and square nozzles are used to improve combustion processes by augmenting heat release, reducing emissions, and improving flame stability. Combustion systems with triangular and square jets (i.e., with corners) benefit from fast bulk mixing between fuel and air, while small-scale turbulence enhances mixing at the molecular level. Increased mixing of the jet plume with the surroundings which decreases jet detection is a key goal in the design of stealth aircraft.

Investigation into the properties of non-circular jets has also been motivated by their enhanced characteristic entrainment properties relative to those of comparable circular jets. ${ }^{2}$ This enhanced entrainment is believed to be mainly the result of self-induced vortex loop deformations. Vortex loops have been suggested as a means for extinguishing gas and oil well fires. The compact nature of vortex loops also makes them ideal as simpler building blocks in the modelling of more complex flows, including axis-symmetric turbulent jets and boundary layers. ${ }^{3}$

Vortex loops are created by the impulsive ejection of a fluid through a pipe or orifice into a quiescent fluid. Although many authors have reported on the behaviour of incompressible vortex loops, ${ }^{4,5}$ at the same time not much work has been carried out on the characteristics of compressible and non-circular vortex loops. This is due to the unsteady and highly-three dimensional nature of the flow. Studies such as: Minota ${ }^{6-8}$ and Baird ${ }^{9}$ have looked into the formation and propagation of circular vortex rings using techniques such as schlieren and interferometry. The PIV results of Arakeri et al. ${ }^{10}$ provides a detailed analysis regarding the propagation, speed, and decay of compressible vortex rings, again circular in nature, at various Mach numbers. Jiang et al. ${ }^{11}$ are of the few authors to provide insight into the nature of formation and propagation of non-circular compressible vortex loops.

The current investigation examines the flowfield induced by the diffraction of a shock wave and the consequent vortex loop generated at the exit of a shock tube. Different exit nozzle geometries were utilised to study shock waves and vortex loops of various geometries over a range of flow Mach numbers. 


\section{EXPERIMENTAL SETUP}

\section{A. The Shock Tube}

Experiments were carried out using a circular shock tube to generate the shock wave and the consequent vortex loop. The inner and outer diameters of the shock tube were $d_{i}=30 \mathrm{~mm}$ and $d_{o}=38 \mathrm{~mm}$, respectively. Driver gas (air) pressures of $P_{4}=4,8$, and $12 b a r$ were examined. The pressure in the driven section $\left(P_{1}\right)$ was atmospheric. An industrial film diaphragm divided the two sections of the shock-tube. The thickness of the diaphragm was chosen to be 23,55 , and $75 \mu \mathrm{m}$, and were chosen for being the minimum thickness which would sustain the desired pressure without spontaneously rupturing. The bursting of the diaphragm was initiated manually with a plunger.

Various adaptors were designed that could be attached to the end of the circular shock tube section. This would allow vortex loops of different shapes to be studied. These include a circular nozzle with an internal diameter of $15 \mathrm{~mm}$, two elliptical nozzles with minor to major axis ratios of $a / b=0.4$ and 0.6 with $b=30 \mathrm{~mm}$, a square nozzle with side lengths of $30 \mathrm{~mm}$, and two exotic nozzles resembling a pair of lips, as shown in Figure 1, with minor to major axes ratios of $a / b=0.2$ and 0.5 with $b=30 \mathrm{~mm}$. All nozzles had a wall thickness of $4 \mathrm{~mm}$, with a gradual area change from the $30 \mathrm{~mm}$ internal diameter circular shock tube section to the various shapes over a length of $300 \mathrm{~mm}$. The length of the circular driven section was $1310.5 \mathrm{~mm}$. The critical length for the circular shock tube section was found to be $12.3 d_{i}, 8.53 d_{i}$, and $7.23 d_{i}$ for $P_{4} / P_{1}=4,8$, and 12 , respectively. Utilising the critical length would produce a pulsed upstream condition where the duration and magnitude of the pulse can be controlled up to the nozzles' inlet. ${ }^{12}$

\section{B. Flow Visualisation Apparatus}

Schlieren ${ }^{13}$ pictures were captured, in time sequence, by delaying the triggering of the light source using a signal synchronisation unit. Figure 2 shows the arrangement of the schlieren system. The schlieren system utilised an Argon stabilised pulsed flash system (625ns duration, 5Joules) as a light source. The light generated by the flash passes through a plano-convex lens $(75 \mathrm{~mm}$ in diameter and $75 \mathrm{~mm}$ focal length) and the converged light spot passes through an iris $(3 \mathrm{~mm}-50 \mathrm{~mm})$ and cut by a slit. Then, the resulting light beam 
reaches a parabolic mirror of $203.3 \mathrm{~mm}$ diameter and $1016 \mathrm{~mm}$ focal length. The collimated light beam passes through the test region and then is focused by another parabolic mirror. The focal point of the mirror is on a knife edge. A transducer placed $660 \mathrm{~mm}$ from the diaphragm along the driven section of the shock tube was used to trigger the flash lamp and to capture a single image. The pressure pulse generated by the passage of the shock wave acted as the trigger. The setup is identical to that used by Kontis et al. ${ }^{14}$

In addition to the high-quality single frame schlieren and shadowgraphy images, the Shimadzu Hyper Vision HPV-1 camera was also utilised to obtain high-speed video photography of the flow field, albeit at a lower resolution. This enabled a better understanding of the transient flow pattern. Images were acquired at a frame rate of $63 \mathrm{Kfps}$ with and exposure time of $4 \mu s$. This required a continuous light source (300W Xenon lamp) instead of the Argon flash lamp used for single exposure schlieren.

\section{Flow Quantification Apparatus}

A TSI high frame rate (1500 frames per second at $1024 \times 1024$ pixels resolution) PIV system consisting of a high repetition rate laser $(10 \mathrm{~mJ}$ at $1 \mathrm{kHz})$ for illumination, with a light arm for easy manipulation of the laser beam and a range of light sheet optics was used. A high frame rate camera (Photron APX RS) was used to capture data in an on-board memory of 8GB. A model 9306A TSI six-jet atomizer was used to generate seeder particles. The particle relaxation time ${ }^{15}$ for a $1 \mu \mathrm{m}$ olive oil particle was calculated as $\tau=2.2 \mu s$. A synchroniser allows control of timing of capture and has the facility for external inputs for triggering the PIV system. A high specification PC with TSI's Insight PIV software installed enables data download and analysis. In addition, TecPlot 10 is also loaded for data display and analysis (with TSI Plot PIV add-on).

The same transducer which was used to trigger the schlieren apparatus was also utilised to act as the trigger for the PIV acquisition. The size of the interrogation zones $(32 \times 32$ pixels $)$ and the timing between the two PIV frames $(\Delta T=3 \mu s)$ was chosen based on the theoretical Mach number of the flow behind the incident shock wave, ${ }^{16}$ and the high-speed schlieren photographs of the flow which were obtained prior to the PIV experiments. The total interrogation area was approximately $80 \times 90 \mathrm{~mm}$. An enclosure was designed which encased the exit of the shock tube. Prior to each run, this enclosure was filled with tracer particles 
along with the driven section of the shock tube.

Two sets of PIV measurements were conducted, the first involved placing the laser sheet parallel to the shock tube in a stream-wise sense as shown schematically in Figure 3. For the second case the laser sheet was placed normal to the shock tube such that the flow is captured head-on as shown in Figure 4 at locations $x=10,25,50,80$, and 110 $\mathrm{mm}$ from the nozzle exit. With the laser sheet parallel to the shock tube the $\omega_{z}$ component of vorticity can be deduced while with the laser sheet normal to the shock tube exit the $\omega_{x}$ component of vorticity is obtained.

\section{RESULTS}

Once the expansion wave from the end of the shock tube driver section arrives at the nozzle exit it will reflect differently from the different nozzles and thus will superimpose different efflux conditions. The flow conditions at the exit of the nozzles in terms of pressure and velocity vary with time, changing for a fixed driver pressure when different nozzle geometries are used. This is due to the different mass flows and transverse waves set up.

The experimental exit shock Mach numbers $M_{s e}$, for the various driver pressures and nozzles used, is calculated from the fluid velocity obtained from the PIV data. These are given in Table I. The flow $R e$ number, $R e=\rho u_{p} L / \mu$, was calculated in terms of the density behind the incident shock $\rho$, the induced velocity behind the incident shock $u_{p}$ obtained from PIV measurements, and the dynamic viscosity $(\mu)$ corresponding to the flow behind the incident shock. The Re numbers are also presented in Table I. The length scale $(L)$ used in the calculation of $R e$ is dependent on the shape of the nozzle employed: for the square nozzle $L=30 \mathrm{~mm}$, for the circular nozzle $L=15 \mathrm{~mm}$, for the elliptic nozzles $L=12 \mathrm{~mm}$ and $L=18 \mathrm{~mm}$ for $a / b=0.4$ and 0.6 , respectively. For the two exotic nozzles $L=6 \mathrm{~mm}$ and $L=15 \mathrm{~mm}$ for $a / b=0.2$ and 0.5 , respectively.

Circulation $\Gamma$ is a measure of the strength of a vortex. From Green's theorem, ${ }^{17}$ the circulation around any finite closed curve $\mathbf{c}$ in the $\mathbf{x}, \mathrm{y}$-plane may be expresses in terms of a surface integral, namely,

$$
\Gamma_{z}=\oiint_{A}\left(\frac{\partial v}{\partial x}-\frac{\partial u}{\partial y}\right) d x d y
$$

However, Arakeri et al. ${ }^{10}$ found that the circulation calculated using Eq.(1) to be less accurate than the circulation found by integrating the velocity components along the curve 
c, given by,

$$
\Gamma_{z}=\oint_{c}(u d x+v d y)
$$

Therefore, for the present analysis the circulation has been computed using Eq.(2). The circuit shown in Figure 5 is used to calculate the circulation. Similar to Arakeri et al., ${ }^{10}$ the curve $\mathbf{c}$ is chosen to lie close to the boundaries of the PIV image so that all the vortex vorticity is contained in the closed contour c.

For the PIV experiments the repeatability is determined by: (i) setting the same driver pressure, (ii) having the same time delay output from the delay generator, and (iii) triggering the laser and camera at two consecutive frames separated by $\Delta T$. The aforementioned procedure was repeated twice and the location of the vortex loop for the two cases was compared frame by frame. The maximum difference between the location of the vortex loop for the sets of repeats was calculated as $1.5 \%$.

\section{A. Circular Nozzle}

According to Moore, ${ }^{18}$ for the range of Mach numbers $0<M<1.58$ the effect of compressibility is to reduce the speed of propagation of a vortex loop. To conserve momentum, this reduction in speed is balanced by the increase in mass of the vortex loop, as evident in the schlieren images of Figure 6 for the two extremum of driver pressures. In Figure 6(b) we also notice the formation of a Mach disk as a result of the supersonic flow exiting the nozzle.

Figure 7 shows the velocity magnitude calculated as: $\sqrt{U^{2}+V^{2}}$. The time, $t$, is given from the instance the shock wave exits the tube. In Figure 7(a) the resultant flow field from the emergence of the shock front and the circular vortex loop is evident. At $t=0.08 \mathrm{~ms}$ in Figure 7(b), the vortex loop has just arrived at the location of the laser sheet which is why the outward component of velocity is significantly less. As the shock front spreads and the vortex loop evolves downstream, the flow field is divided into two segments visible as the two concentric rings in Figures $7(\mathrm{c})$ and $7(\mathrm{~d})$. The outer ring with the vectors moving radially outwards is induced mainly by the passage of the shock wave. The inner ring with the slightly higher velocity magnitude and the vectors moving inwards results from the entrainment of surrounding fluid.

If we move further away from the nozzle where the vortex loop has had more time to 
develop, we can clearly see in the results of Figure 8, especially Figures 8(b) and 8(c), the extreme velocity imposed by the approaching vortex loop.

The circular vortex loop exhibits distinct changes in diameter for each shock tube driver pressure, displayed in Figure 9(a). The diameter of the vortex loop, $D_{r}$, is nondimensionalised with reference to the internal diameter of the nozzle, $d_{i}$. The dimensionless time $\left(t-t_{1}\right) \times U_{s} / d_{i}$, is based on the specified time $t$, the time of the initial emergence of the vortex loop $t_{1}$, the corresponding shock wave velocity $U_{s}$, and the internal diameter of the nozzle. Uncertainty estimation given by Holman ${ }^{19}$ has been used to determine the accuracy, in the form of error bars. For all driver pressures, $P_{4}$, the diameter of the vortex loop initially increases but finally reaches a uniform value. Because the flow is viscous and not barotropic, Kelvin's Theorem does not hold $(D \Gamma / D t \neq 0)$ as clearly seen in Figure $9(\mathrm{~b})$. The vortex loop circulation displays a dependence on the diameter. This will be further discussed when examining the vortex loop with axis ratio of $a / b=0.6$.

\section{B. Elliptic Nozzle $a / b=0.4$}

Vortex loops go through four stages of development: (1) generation or formation, (2) stable laminar phase, (3) unstable or wavy phase, and (4) turbulent phase. ${ }^{20}$ Whether the vortex loop goes through all stages depends on the initial conditions during the generation phase. Figure 10 compares the elliptic vortex loops generated at different flow Mach numbers at approximately the same distance away from the nozzle. Since there is a change in curvature of the generating nozzle, the vortex loop translational velocity varies along its length. Figure 10(a) has the characteristics of the stable laminar phase where the vortex filament is clearly visible and instability-free. The vortex filament in Figure 10(b) is not as distinct due to the increased entrainment of ambient fluid. In the lower half of the vortex loop we can see the bending and waviness to be present which shows the onset of the unstable phase. Finally, in Figure 10(c) the vortex loop is accompanied by intense acoustic waves along with a much larger entrainment body and a very turbulent trailing jet. This figure demonstrates that by changing the flow Mach number, different stages of vortex loop development can be observed without the need of tracing one particular vortex loop throughout its life cycle for a specific Mach number.

At high Mach numbers, due to compressibility effects, the flow is dramatically changed 
and becomes quite complicated. Unlike the lower driver pressure case of Figure 10(a) where the flow behind the main vortex loop is almost correctly expanded, the flow in Figure 10(c) has characteristics of an underexpanded jet with the shock cells becoming larger. For nonaxisymmetric jets the level underexpansion can be different for the same jet when viewed from different directions due to the complex jet geometry and the nozzle aspect ratio. ${ }^{21-23}$

The artwork of Figure 11 illustrates the motion of the elliptic vortex loop. From the velocity traces of the head-on view presented in Figure 12, the axis switching can easily be predicted. Axis switching refers to the state where the minor and major axes of the vortex loop are interchanged. This phenomenon will be dealt in detail when considering the square vortex loop. Since the largest induced velocity magnitudes occur on the upper and lower portions, this stretches the vortex loop vertically. The faster growth rate of the jet width along the minor axis of the nozzle causes this behaviour. In Figure 12(b) due to the elongation of the jet boundary along the minor axis, the width along the minor axis exceeds that along the major axis. This behaviour is also apparent at higher flow Mach number shown in Figure 13. From the upstream flow of Figure 13(c) we can ascertain the flow downstream. The velocity contours show that the flow is being compressed vertically, implying that the vortex loop downstream is taking the shape it originally had when leaving the nozzle.

In Figure 14 the colours present the vorticity profile calculated as $\omega_{z}=\frac{\partial u}{\partial y}-\frac{\partial v}{\partial x}$, with the vectors showing the velocity magnitude where the length of the vectors is proportional to the magnitude of the velocity. The $x$ - axis is measured by the software from the edge of the raw PIV image recorded by the camera. The interrogation zones were chosen to start $\approx 5 \mathrm{~mm}$ from the nozzle exit to avoid any reflections from the nozzle interfering with the signal from the tracer particles. At the lower diaphragm pressure ratio presented in Figure 14(a), the vorticity is spread more in the transverse direction whereas due to the high speed flow exiting the tube at $P_{4} / P_{1}=12$ in Figure $14(\mathrm{~b})$, the vorticity is contained to the region surrounding the cores with the maximum velocity occurring between the two cores. This shows that compressibility reduces the jet spreading rate.

With increasing flow Mach number, the slipstream increases its angle relative to the horizontal. This causes the diameter of the vortex loop to be innately larger at higher shock tube driver pressures, as shown in Figure 15(a). The presence of viscous effects causes a diffusion of vorticity into or out of the fluid circuit, and consequently changes the circulation 
as evident in Figure 15(b). ${ }^{24}$

\section{Elliptic Nozzle $a / b=0.6$}

From the schlieren images of Figure 16 it is apparent that the difference in translational velocity of the different parts of the larger vortex loop are not as prominent compared the smaller vortex loop of $a / b=0.4$. At high flow Mach numbers an embedded shock is formed within the core of the vortex loop which translates downstream along with the vortex loop. It is a rearward facing shock and is generated due to the supersonic flow in the recirculating region in the frame of reference of the vortex loop.

The head-on views of Figures 17(a) and 17(b), taken 25mm from the nozzle exit, show an axis-symmetric profile since the jet widths along the minor and major axes are almost identical. This is because with the current nozzle axis ratio, we are quite close to a circular nozzle in which all parts of the loop travel downstream at the same velocity. However, as the vortex loop travels downstream, its upstream velocity profile indicates that the vortex loop is being stretched vertically, i.e., going through the process of axis switching.

The time scale of the rolling up of the vortex sheet is larger at lower driver pressures. This leads to a thicker vortex core. At high flow Mach numbers vortices may develop 'vacuum cores' if the strength of the vortex is sufficient to centrifuge gas away from the centreline. This results in the tracer particles being thrown out of the core as evident in Figure 18. Although this phenomenon prevents us from obtaining quantitative data from the core, it is an indication of the relative strength of the vortex circulation. In the same figure we notice the presence of vorticity upstream of the primary vortex loop. As vorticity is dumped into the wake, its magnitude within the primary vortex loop gradually decreases.

From the results of Figure 19 the relation between vortex loop diameter and circulation is readily seen. From conservation of angular momentum, the circulation increases due to the increase of vortex loop diameter and the consequent stretching of the vorticity lines given by the first term on the right hand side of Eq. (3). ${ }^{3}$

$$
\frac{D \omega}{D t}=\omega \cdot \nabla u+\nu \nabla^{2} \omega
$$

The total mass of the vortex loop increases due to the entrainment of ambient fluid. In order to conserve momentum, the translational velocity of the vortex loop decreases. Since 
the translational velocity is dependent on the circulation, hence the circulation must also tend to decrease.

\section{Square Nozzle}

Initially as the planar incident shock exits the tube it adopts the square shape of the nozzle, discerned from Figure 20. What follows the shock wave is the square vortex loop leaving the tube, created due to baroclinic effects. ${ }^{25}$

Figure 21 shows the vortex loop for the case of $P_{4} / P_{1}=4$. Following the motion of the vortex loop carefully we observed that the corner portions move ahead, while the remaining side portions develop a higher curvature and with this high curvature they start moving with an increased velocity until they arrive at the location of the corners and the loop becomes planar. Along with this alignment comes the process of axis switching, which is well known for incompressible jets. Figure 22 depicts the motion of the square vortex loop and the process of axis switching. The underlying mechanism for this process will be addressed later. As the vortex loop evolves downstream, it develops azimuthal disturbances. At higher flow Mach numbers the axis switching is less apparent since the vortex loop develops these instabilities much faster.

Figures 23(a) to 23(c) show the velocity magnitude, and Figure 23(d) is the vorticity

profile calculated as $\omega_{x}=\frac{\partial w}{\partial y}-\frac{\partial v}{\partial z}$. All profiles were taken $50 \mathrm{~mm}$ from the shock tube exit. The induced velocity is a combination of the motion of the shock wave and the approaching vortex loop (Figure 23(b)). The velocity is zero at the potential core since the velocity component is normal to field of view. It is interesting to observe the extremely high velocities induced at the corners in Figure 23(c). The vorticity profile of Figure 23(c) presented in Figure 23(d) shows the presence of out-flow vortex pairs at the corners which are responsible for this increased velocity.

Further away from the nozzle where the vortex loop develops azimuthal undulations, its consequent shape is reflected in the induced velocity downstream, as visible in Figure 24(a). Even $80 \mathrm{~mm}$ away from the nozzle, the effect of the vortex pairs mentioned earlier is still ongoing, and causes an increased expansion of the flow at the corner regions of Figure 24(b). As the vortex loop moves downstream, the induced flow behind it, shown in Figure 24(c), shows the location of maximum induced velocity has switched from the corners to 
the flat sides. Because the portions of the vortex loop aligned with the corners move faster downstream, the portions aligned with sides of the nozzle are relatively closer to the laser sheet and hence induce a higher velocity at these regions.

Figure 25 examines the behaviour of the vortex pairs generated at the nozzle exit in more detail. Ahead of the primary vortex loop, aligned with the nozzle corners, two vortices are generated which induce an outwards flow (Figure 25(a)). As the primary vortex loop progresses downstream, each vortex pairs-up with its adjacent neighbour and due to mutual induction accelerate the flow inwards (Figure 25(b)). It is the motion of these vortices which give rise to the process of axis switching.

Figure 26 shows the change in diameter and circulation of the square vortex loop over time. The diameter of the vortex loop, $D_{r}$, is non-dimensionalised with reference to the side length of the nozzle, $d_{i}$ The diameter of the vortex loop increases quite rapidly during the first segment of the vortex loop travel for all shock tube driver pressures. The diameter then reaches an asymptotic value before increasing again. Similar to the previous vortex loops, the dependence between vortex loop diameter and circulation is also present.

\section{E. Exotic Nozzle $a / b=0.2$}

A common feature which was observed for this particular nozzle for the various diaphragm pressure ratios, is the dramatic reduction of velocity in the vicinity of the singular corners. The is readily observed in the head-on view of Figure 27.

From the sequence of schlieren photographs of Figure 28 it is clear that the turbulent vortex loop created at higher values of driver pressure develops a much larger entrainment region. This is reflected in the vorticity distribution presented in Figure 29. In Figure 29(a) the vorticity which is deposited into the wake is also captured by the PIV measurements.

The rate of growth of the vortex loop diameter is almost independent of flow Mach number for the early stages of its propagation (Figure 30(a)). The circulation increases due to the vortex loop growing in size. As the vortex loop propagates downstream it begins to shed vorticity into its wake. Therefore, no matter how much the diameter continues to increase, due to effects of viscosity, the total circulation reduces (Figure 30(b)). 


\section{F. Exotic Nozzle $a / b=0.5$}

With change in nozzle aspect ratio from $a / b=0.2$ to 0.5 , the flow is qualitatively similar. The reduced flow expansion occurring at the singular corners of the nozzle is still present, as it is shown in Figure 31, for the vortex loop approaching and vacating the location of the laser sheet.

Due to the relatively high Reynolds number of the flow, the vortex loop appears turbulent immediately upon ejection from the nozzle, this is discerned from Figure 32. The interaction between the vortex and the embedded shock present within the vortex loop gives rise to the shocklets visible above and below the vortex cores (Figure 32(b)). This occurs from the shock splitting phenomenon. ${ }^{26}$ The turbulent characteristic of the vortex loop leads to a rapid growth rate of the vortex diameter and circulation visible in Figure 33. As the nozzle aspect ratio increases, the diameter of the vortex loop is affected more by the initial flow Mach number. This is readily seen when comparing Figures 33(a) and that of Figure 30(a). The same behaviour is observed when comparing the two elliptical vortex loops.

Similar to the two elliptical vortex loops in which the difference in translational velocity of the different portions of the loops is less pronounced for the larger loop, the exotic vortex loop with a larger minor to major axis ratio shows less pronounced velocity variations along its periphery, compared to its smaller counterpart.

Figures 34 and 35 provide details of the same initial flow conditions $\left(P_{4} / P_{1}=8\right)$ obtained at different times, but they are presented separately so that the contour levels can be adjusted to better identify key flow features. As the vortex loop approaches, four distinct regions of high velocity are visible in the PIV results of Figure 34. The physical properties of flows through such nozzles and their underlying mechanism are not fully understood at this stage. The increased velocity components in the vertical direction of Figure 35(a) are indicative of the vortex loop going through the process of axis switching. Although the greatest magnitude of velocities occur upstream of the vortex loop in the side portions (Figure 35(b)), after a short period this behaviour is altered and the maximum induced velocities occur in the upper and lower portions of the flow (Figure 35(c)). This indicates that the vortex loop downstream is being compressed in the vertical direction. 


\section{G. Vortex Loop Propagation Velocity}

The initial translational velocity of a vortex loop $U_{T}$ is related to the circulation $\Gamma$, the radius of the vortex loop $R$, and the radius of the vortex loop core $R_{c}$, by the relation:

$$
U_{T}=\frac{\Gamma}{4 \pi R}\left[\ln \left(\frac{8 R}{R_{c}}\right)-0.558+I_{\text {comp }}\right] .
$$

Omitting the last term in the bracket, $I_{\text {comp }}$, the expression provides the translational velocity for an inviscid incompressible vortex loop with a circular core. ${ }^{27}$ The term $I_{\text {comp }}$, is the correction factor provided by Moore ${ }^{18}$ for the effects of compressibility.

Equation (4) depicts the motion of a circular vortex loop. Having a circular vortex loop essentially means that the if we take a vertical slice through the vortex loop, the two vortex cores which are seen are symmetrical about the horizontal plane. This is readily seen in Figure 36(a). If we take the same vertical slice through, say, an elliptic vortex loop, we are again left with two vortex cores on the top and bottom which are circular in crosssection, visible in Figure 36(b). Table II provides theoretical and experimental vortex loop translation velocities, non-dimensionalised with respect to the corresponding shock velocities $U_{s}$. The experimental values correspond to the initial phase of vortex loop propagation. At this stage, the vortex core is still circular and effects of viscosity are minimal.

The disagreement between theory and experiments is mostly due to the neglect of viscosity and the non-uniform vortex core cross-section, since compressibility effects have been accounted for. As mentioned earlier, the type of vortex loop depends strongly on the initial conditions of formation. At high Re numbers, the vortex loop starts off turbulent with greater entrainment of ambient fluid. This indicates higher effects of viscosity.

\section{CONCLUSIONS}

The present study involved the shock wave and consequent vortex loop generated when a shock tube with various exit nozzle geometries is employed. These include: a $15 \mathrm{~mm}$ diameter circular nozzle, two elliptical nozzles with minor to major axis ratios of $a / b=0.4$ and 0.6 , a $30 \times 30 \mathrm{~mm}$ square nozzle, and two exotic nozzles resembling a pair of lips with minor to major axis ratios of $a / b=0.2$ and 0.5 . The experiments were performed for driver gas (air) pressures of $P_{4}=4,8$, and 12bar; with the pressure in the driven section being 
atmospheric. Using high-speed schlieren photography along with PIV analysis of the streamwise and head-on flows, the formation and propagation of the different types of shock waves and vortex loops generated were examined.

The results showed that similar to incompressible jets, upon the introduction of any corners, such as the square or elliptic cases, the vortex loop propagation becomes nonuniform along the entire length of the loop. The parts generated at the corners travel faster downstream while the portions generated by the flat sides or corners with less curvature travel faster in the transverse direction.

From the head-on velocity data obtained through PIV measurements, it is clear that from the induced flow downstream and upstream of the vortex loop, it is possible to predict the motion of vortex loops. The presence of vortex pairs, generated at the nozzle exit upon formation of the main vortex loop, plays an important part in the motion of compressible vortex loops. The motion of the main vortex loop is as a result of the interaction of these vortex pairs.

The circular vortex loop exhibits quite distinct changes in diameter for each shock tube driver pressure. This is partly because the diameter of the non axis-symmetric vortex loops were based on the schlieren photographs of only one perspective. This does not take into account how the diameter of the vortex loop varies when viewed from a different plane. The circulation of the circular vortex loop is also the highest compared to the other vortices examined. Again, due to the three dimensionality of the other loops. Because of the constant acceleration and deceleration of some parts of the non-axisymmetric vortex loops, their induced circulation is relatively less.

The results indicate that the introduction of singular corners, (corners where the two sides meet at infinity), the expansion of the flow is dramatically decreased. However, vortex loops generated from these nozzle still show the dependency of translational velocity to the radius of curvature of the nozzle. More research is underway to compare the findings of the present study to existing numerical and analytical techniques and provide more insight into the physical properties of such flows.

To predict the rate of propagation of vortex loops, more information relating to the structure of the vortex core and the distribution of flow properties is required at higher Re numbers. Extreme flow conditions within the core, exacerbates the ability to obtain non-intrusive quantitative data. 


\section{Acknowledgments}

The authors are indebted to the technical staff at The University of Manchester for their assistance, and for the help and advice of Dr. Martin Hyde (TSI) for the installation and setup of the PIV system. The support of the EPSRC Engineering Instrument Pool especially Mr. Adrian Walker, for the loan of the PIV and the Shimadzu systems as well as the financial support of the EPSRC are greatly acknowledged. The authors would also like to thank Mr. David Shawley of HiFi Industrial Film for supplying the diaphragm sheets, and Mr. Craig Hale for help in the preparation of the artwork. 
1 Gutmark, E.J., Grinstein, F.F., "Flow control with noncircular jets," Annual Review of Fluid Mechanics 31, pp. 239-272, (1999).

2 Yoon, J. H., Lee, S.J., "Investigation of the near-field structure of an elliptic jet using stereoscopic particle image velocimetry," Measurement Science and Technology 14, pp. 2034-2046, (2003).

3 Green, S. I., "Fluid Vortices," Kluwer Academic Publishers, (1995).

${ }^{4}$ Oshima, Y., Izutsu, N., Oshima, K., Hussain, A. K. M. F., "Bifurcation of an elliptic vortex ring," Fluid Dynamics Research 3, pp. 133-139, (1988).

${ }^{5}$ Grinstein, F. F., DeVore, C. R., "Dynamics of coherent structures and transition to turbulence in free square jets," Physics of Fluids 8, pp. 1237-1251, (1996).

6 Minota, T., "Interaction of a shock wave with a high-speed vortex ring," Fluid Dynamics Research 12, pp. 335-342, (1993).

7 Minota, T., "Dynamic motion of a compressible vortex ring," Proceedings of SPIE-the international society for optical engineering 3173, pp. 241-248 (1997).

8 Minota, T., Nishida, M., Lee, M. G., "Shock formation by compressible vortex ring impinging on a wall," Fluid Dynamics Research 21, pp. 139-157, (1997).

9 Baird, J.P., "Supersonic vortex rings," Proceedings of Royal Society of London A 409, pp. $59-65,(1987)$.

10 Arakeri, J. H., Das, D., Krothapalli, A., Lourenco, L., "Vortex ring formation at the open end of a shock tube: A particle image velocimetry study," Physics of Fluids 16, pp. 1008-1019, (2004).

11 Jiang, Z., Onodera, O., Takayama, K., "Evolution of shock waves and the primary vortex loop discharged from a square cross-section tube," Shock Waves 9, pp. 1-10, (1999).

12 Brouillette, M., Hebert, C., "Propagation and interaction of shock-generated vortices," Fluid Dynamics Research 21, pp. 159-169, (1997).

13 Merzkirch, W., "Flow visualization," Academic Press Inc, (1974).

14 Kontis, K., An, R., Edwards, J. A., "Compressible vortex-ring interaction studies with a number of generic body configurations," AIAA Journal 44, pp. 2962-2978, (2006).

15 Melling, A., "Tracer particles and seeding for particle image velocimetry," Measurement Science 
and Technology 8, pp. 1406-1416, (1997).

16 Anderson, J. D., "Modern Compressible Flow, with Historical Perspective," McGraw-Hill, Inc, ed. 2 , (1990).

17 Shapiro, A. H., "The dynamics and thermodynamics of compressible fluid flow, Vol. 1," The Ronald Press Company, (1953).

18 Moore, D. W., "The effect of compressibility on the speed of propagation of a vortex ring," Proceedings of the Royal Society of London A 397, pp. 87-97, (1985).

19 Holman, J. P., "Experimental Methods for Engineers," McGraw-Hill, Inc, (1994).

20 Auerbach, D., "Stirring properties of vortex rings," Physics of Fluids A3, pp. 1351-1355, (1991).

21 Menon, N., Skews, B. W., "Effect of nozzle inlet geometry on underexpanded superonic jet characteristics," 26th International Symposium on Shock Waves (ISSW26), Göttingen, Paper 1020, (2008).

22 Menon, N., Skews, B. W., "Rectangular underexpanded gas jets: effect of pressure ratio, aspect ratio and Mach number," 26th International Symposium on Shock Waves (ISSW26), Göttingen, Paper 1020, (2008).

23 Tsutsumi, S., Teramoto, S., Yamaguchi, K., Nagashima, T., "Structure of underexpanded jets from square nozzles," AIAA 44, pp. 1287-1291, (2006).

24 Kundu, P. K., Cohen, I. M., "Fluid Mechanics," Academic Press, (2004).

25 Takayama, K., Inoue, O., "Shock wave diffraction over a 90 degree sharp corner - posters presented at 18th ISSW," Shock Waves 1, pp. 301-312, (1991).

26 Abate, G., Shyy, W., "Dynamic structure of confined shocks undergoing sudden expansion," Progress in Aerospace Sciences 38, pp. 23-42, (2002).

27 Inoue, O., Takahashi, Y., "Successive generation of sounds by shock-strong vortex interaction," Physics of Fluids 12, pp. 3229-3234, (2000). 
TABLE I: Experimental conditions corresponding to the various nozzles.

\begin{tabular}{|c|c|c|c|}
\hline & $P_{4}[\mathrm{bar}]$ & $M_{s e}$ & $\operatorname{Re}\left(\times 10^{5}\right)$ \\
\hline \multirow{3}{*}{ Ellipse 0.4} & 4 & 1.10 & 0.5 \\
\hline & 8 & 1.32 & 1.7 \\
\hline & 12 & 1.41 & 2.4 \\
\hline \multirow{3}{*}{ Ellipse 0.6} & 4 & 1.09 & 0.7 \\
\hline & 8 & 1.22 & 1.8 \\
\hline & 12 & 1.37 & 3.2 \\
\hline \multirow{3}{*}{ Circle } & 4 & 1.17 & 1.1 \\
\hline & 8 & 1.58 & 4.6 \\
\hline & 12 & 1.62 & 4.9 \\
\hline \multirow{3}{*}{ Square } & 4 & 1.05 & 0.6 \\
\hline & 8 & 1.21 & 2.8 \\
\hline & 12 & 1.43 & 6.3 \\
\hline \multirow{3}{*}{ Exotic 0.2} & 4 & 1.12 & 0.8 \\
\hline & 8 & 1.24 & 3.5 \\
\hline & 12 & 1.35 & 4.1 \\
\hline \multirow{3}{*}{ Exotic 0.5} & 4 & 1.16 & 1.1 \\
\hline & 8 & 1.31 & 3.7 \\
\hline & 12 & 1.51 & 4.6 \\
\hline
\end{tabular}


TABLE II: Vortex loop propagation velocity.

\begin{tabular}{|c|c|c|c|}
\hline & $M_{s e}$ & $\begin{array}{l}U_{T} / U_{s} \\
\text { Theory }\end{array}$ & $\begin{array}{c}U_{T} / U_{s} \\
\text { Exp. }\end{array}$ \\
\hline \multirow{3}{*}{ Ellipse 0.4} & 1.10 & 0.16 & 0.29 \\
\hline & 1.32 & 0.22 & 0.37 \\
\hline & 1.41 & 0.32 & 0.44 \\
\hline \multirow{3}{*}{ Ellipse 0.6} & 1.09 & 0.15 & 0.24 \\
\hline & 1.22 & 0.32 & 0.37 \\
\hline & 1.37 & 0.43 & 0.36 \\
\hline \multirow{3}{*}{ Circle } & 1.17 & 0.4 & 0.52 \\
\hline & 1.58 & 0.67 & 0.59 \\
\hline & 1.62 & 0.98 & 0.59 \\
\hline \multirow{3}{*}{ Square } & 1.05 & 0.19 & 0.26 \\
\hline & 1.21 & 0.47 & 0.31 \\
\hline & 1.43 & 0.45 & 0.39 \\
\hline \multirow{3}{*}{ Exotic 0.2} & 1.12 & 0.16 & 0.21 \\
\hline & 1.24 & 0.17 & 0.42 \\
\hline & 1.35 & 0.25 & 0.48 \\
\hline \multirow{3}{*}{ Exotic 0.5} & 1.16 & 0.13 & 0.18 \\
\hline & 1.31 & 0.31 & 0.44 \\
\hline & 1.51 & 0.32 & 0.43 \\
\hline
\end{tabular}




\section{List of Figures}

1 Cross section of exotic nozzles, $a / b=$ (a) 0.2 (b) $0.5 \ldots \ldots \ldots$

2 Schematic diagram of the schlieren photography setup. . . . . . . . . . . . 22

3 Schematic of PIV setup. . . . . . . . . . . . . . . . . 23

4 Schematic of the PIV setup measuring the head-on flow. . . . . . . . . . . . 23

5 Circuit for calculating circulation. . . . . . . . . . . . . . . . 23

6 Circular nozzle, side view, $P_{4} / P_{1}=$ (a) 4 , (b) $12 \ldots \ldots \ldots \ldots$

$7 \quad$ Head-on PIV results for circular nozzle, $10 \mathrm{~mm}$ from nozzle exit, $P_{4} / P_{1}=12 . \quad 24$

8 Head-on PIV results for circular nozzle, $25 \mathrm{~mm}$ from nozzle exit, $P_{4} / P_{1}=12 . \quad 25$

9 Circular nozzle variation of: (a) vortex loop diameter, (b) vortex loop circulation. . . . . . . . . . . . . . . . . . . 25

10 Elliptic nozzle (0.4), side view, major axis horizontal, $P_{4} / P_{1}=$ (a) 4 , (b) 8 ,

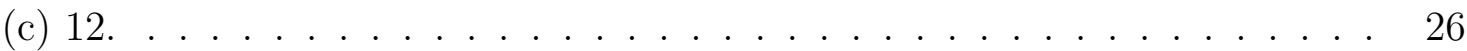

11 Form and direction of motion of the individual parts of elliptic vortex loop. . 26

12 Head-on PIV results for elliptic nozzle (0.4), 50mm from nozzle exit, $P_{4} / P_{1}=4.26$

13 Head-on PIV results for elliptic nozzle (0.4), 25 $\mathrm{mm}$ from nozzle exit, $P_{4} / P_{1}=$

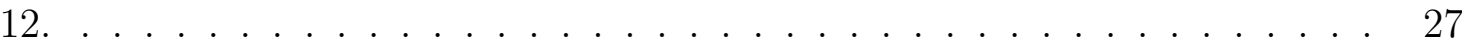

14 PIV results for elliptic nozzle (0.4), side view, major axis horizontal, $P_{4} / P_{1}=$ (a) 4 , (b) 12 .

15 Elliptic nozzle (0.4) variation of: (a) vortex loop diameter, (b) vortex loop circulation. . . . . . . . . . . . . . . . . . . . . 28

16 Elliptic nozzle (0.6), side view, major axis horizontal, $P_{4} / P_{1}=($ a) 4 , (b) $12 . \quad 28$

17 Head-on PIV results for elliptic nozzle (0.6), $25 \mathrm{~mm}$ from nozzle exit, $P_{4} / P_{1}=8$. 29

18 PIV results for elliptic nozzle (0.6), side view, major axis horizontal, $P_{4} / P_{1}=$ (a) 4 , (b) 8 .

19 Elliptic nozzle (0.6) variation of: (a) vortex loop diameter, (b) vortex loop circulation.

20 Square nozzle, $P_{4} / P_{1}=12$, (a) oblique view, (b) oblique view with nozzle rotated (captured using the Shimadzu camera) . . . . . . . . . . . . . . . 31

21 Square nozzle, side view, $P_{4} / P_{1}=4 \ldots \ldots \ldots \ldots$. . . . . . . . . 31

22 Form and direction of motion of the individual parts of square vortex loop. . 31 
23 Head-on PIV results for square nozzle, $50 \mathrm{~mm}$ from nozzle exit, $P_{4} / P_{1}=4 . \quad$. 32

24 Head-on PIV results for square nozzle, $80 \mathrm{~mm}$ from nozzle exit, $P_{4} / P_{1}=4$. . 33

25 Head-on PIV results for square nozzle, $10 \mathrm{~mm}$ from nozzle exit, $P_{4} / P_{1}=8$. . 33

26 Square nozzle variation of: (a) vortex loop diameter, (b) vortex loop circulation. 34

27 Head-on PIV result for exotic nozzle (0.2), $10 \mathrm{~mm}$ from nozzle exit, $P_{4} / P_{1}=8.34$

28 Exotic nozzle (0.2), side view, major axis horizontal, $P_{4} / P_{1}=$ (a) 4 , (b) 12 . . 34

29 PIV results for exotic nozzle (0.2), side view, major axis horizontal, $P_{4} / P_{1}=$

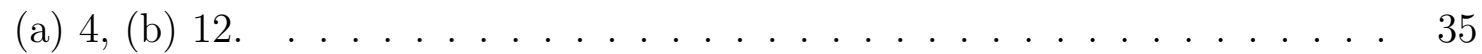

30 Exotic nozzle (0.2) variation of: (a) vortex loop diameter, (b) vortex loop circulation. . . . . . . . . . . . . . . . . . 35

31 Head-on PIV results for exotic nozzle (0.5), $10 \mathrm{~mm}$ from nozzle exit, $P_{4} / P_{1}=8.36$

32 Exotic nozzle (0.5), side view, major axis horizontal, $P_{4} / P_{1}=(\mathrm{a}) 4$, (b) 12 . 36

33 Exotic nozzle (0.5) variation of: (a) vortex loop diameter, (b) vortex loop circulation. . . . . . . . . . . . . . . . . . 37

34 Head-on PIV results for exotic nozzle (0.5), $25 \mathrm{~mm}$ from nozzle exit, $P_{4} / P_{1}=8$. 37

35 Head-on PIV results for exotic nozzle (0.5), $25 \mathrm{~mm}$ from nozzle exit, $P_{4} / P_{1}=8.38$

36 Laser induced fluorescence: (a) circular vortex loop, (b) elliptic vortex loop

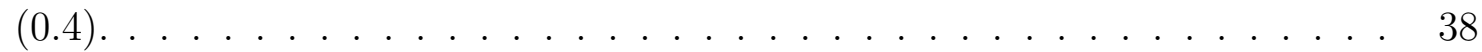




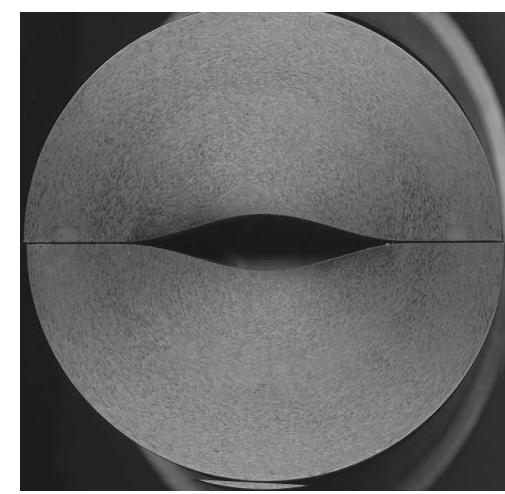

(a)

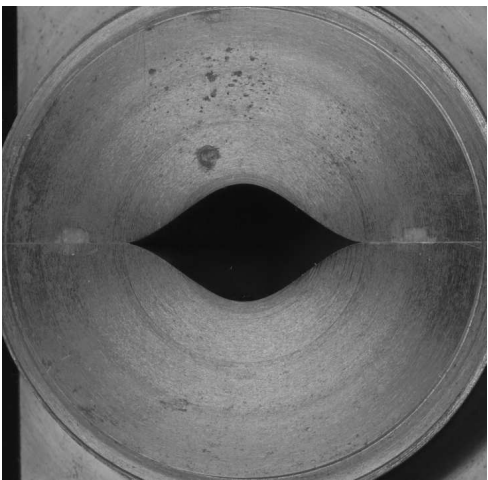

(b)

FIG. 1: Cross section of exotic nozzles, $a / b=$ (a) 0.2 (b) 0.5 .

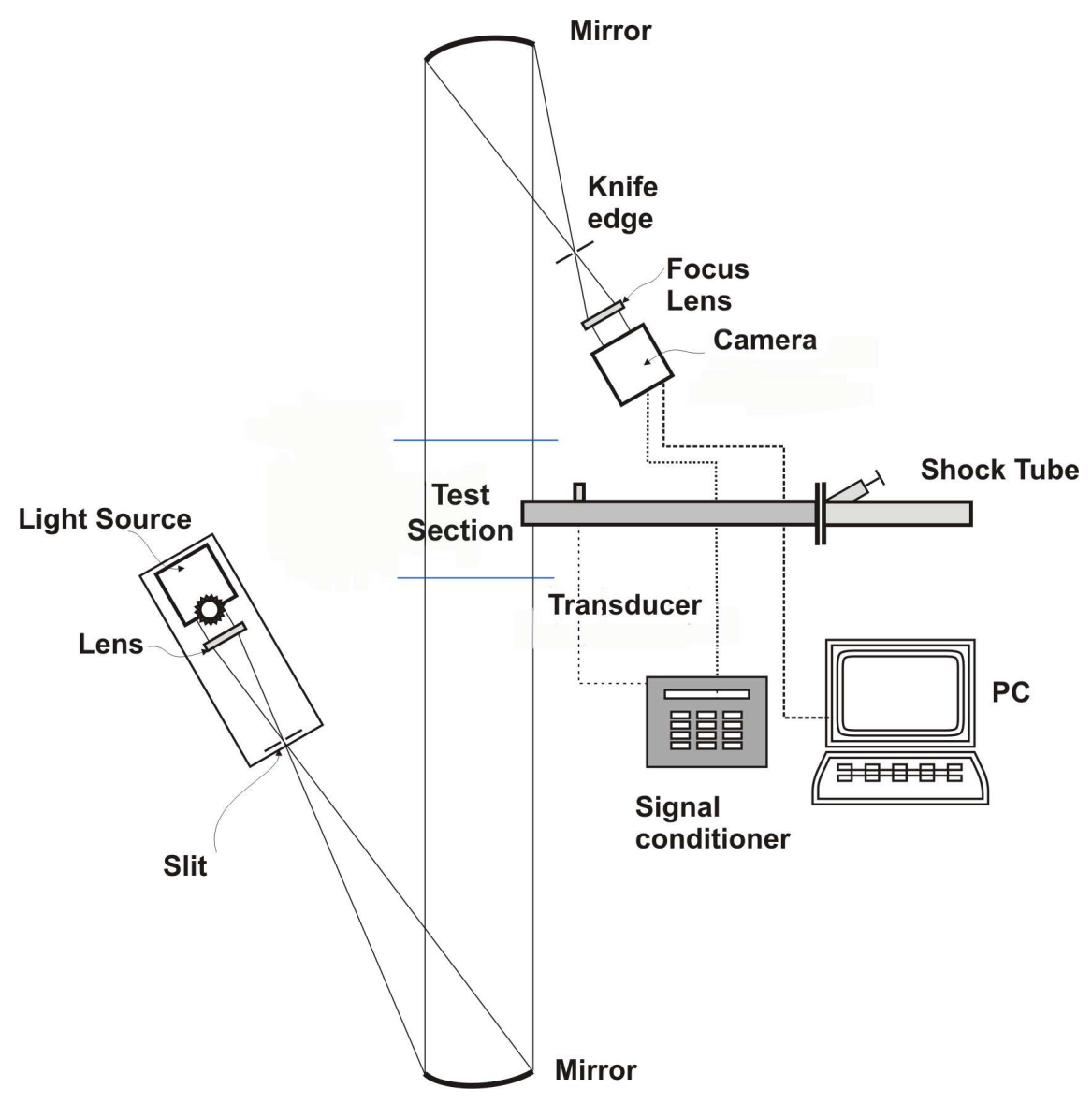

FIG. 2: Schematic diagram of the schlieren photography setup. 


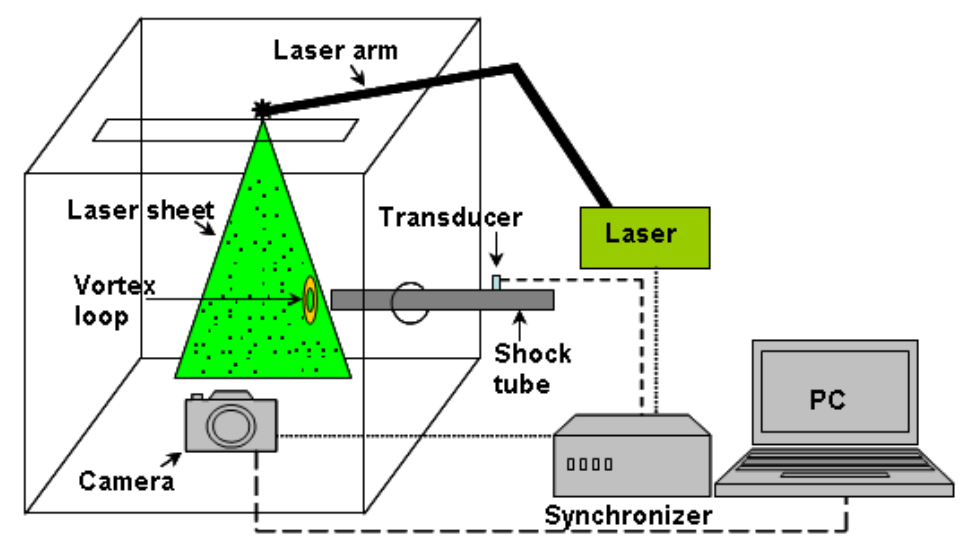

FIG. 3: Schematic of PIV setup.

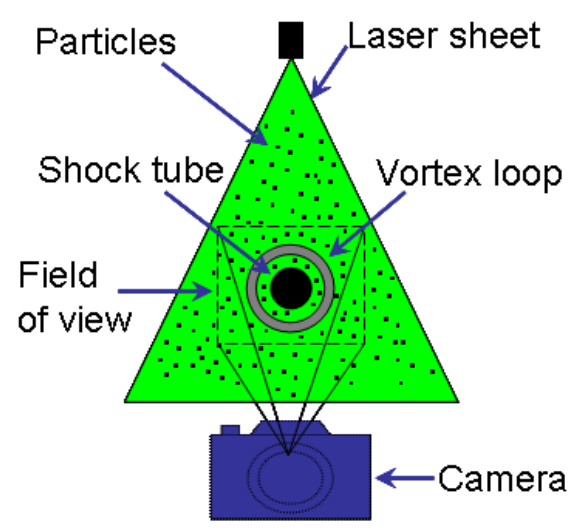

FIG. 4: Schematic of the PIV setup measuring the head-on flow.

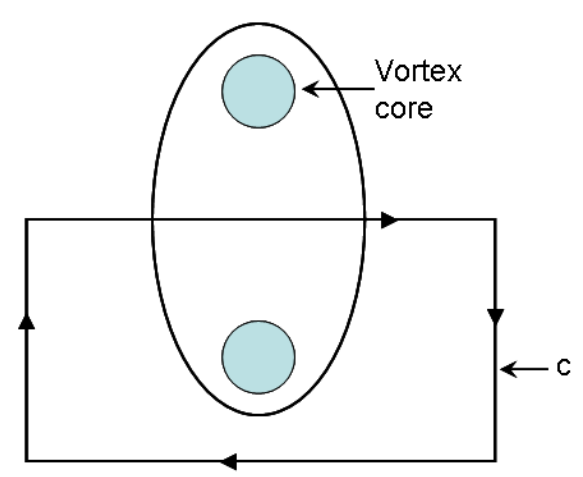

FIG. 5: Circuit for calculating circulation. 


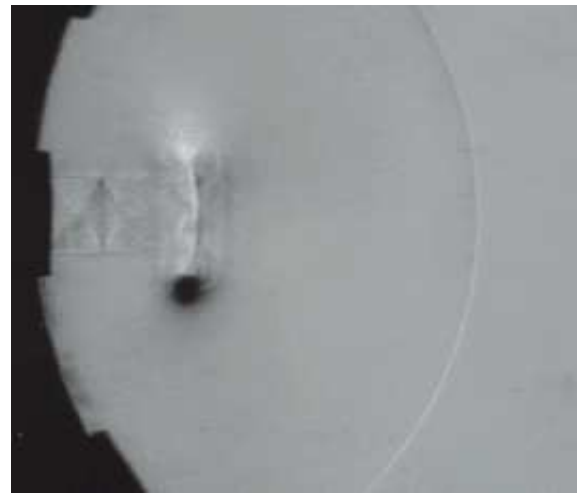

(a)

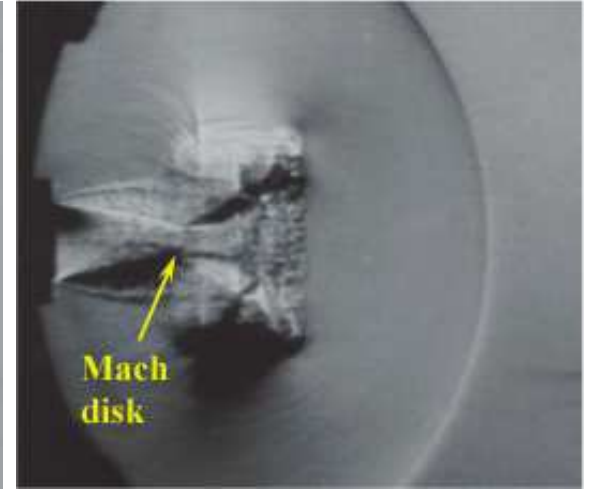

(b)

FIG. 6: Circular nozzle, side view, $P_{4} / P_{1}=$ (a) 4 , (b) 12 .
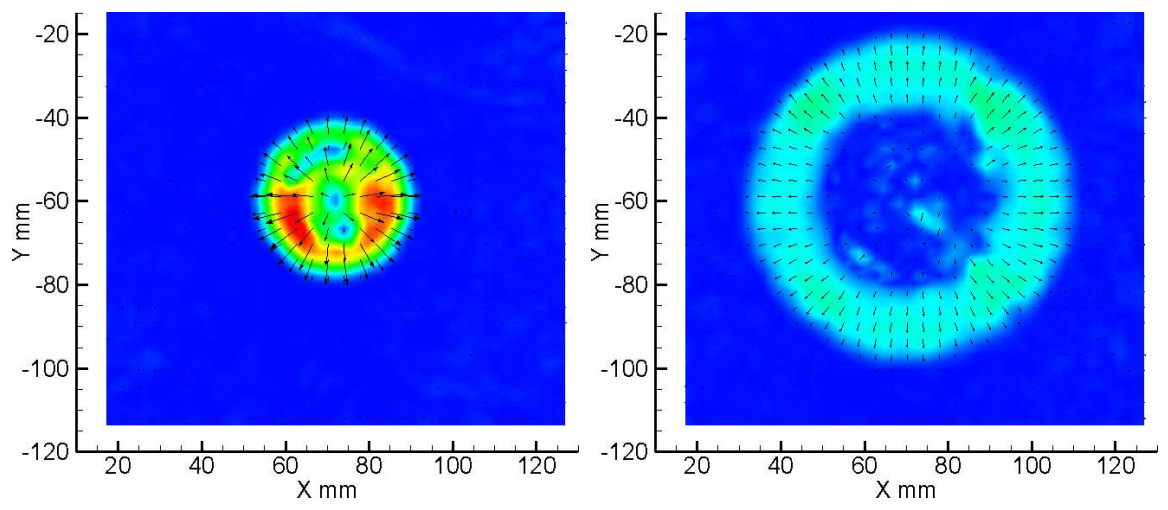

(a) $t=0.05 \mathrm{~ms}$

(b) $t=0.08 \mathrm{~ms}$
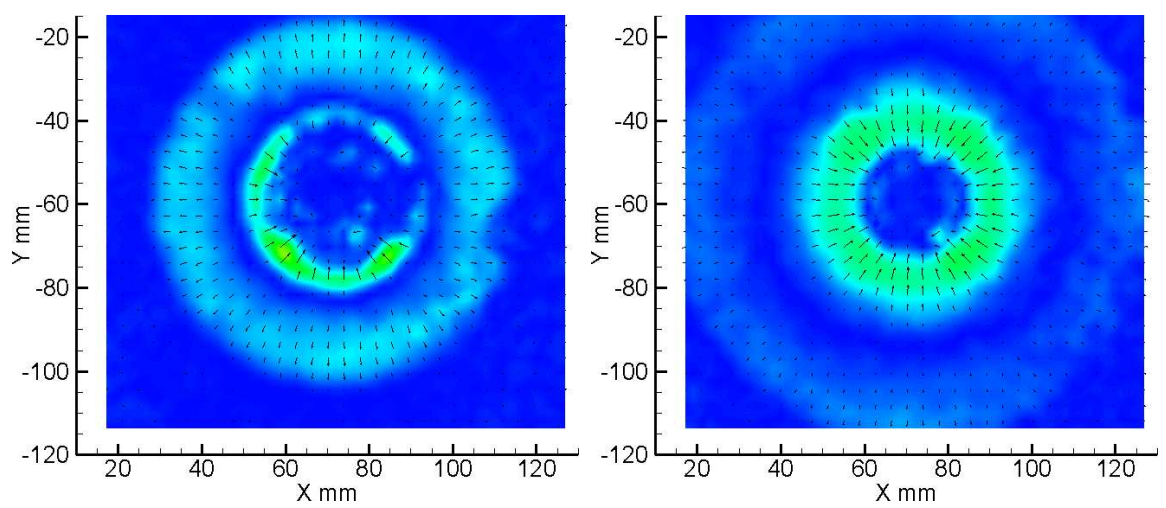

(c) $t=0.12 \mathrm{~ms}$

(d) $t=0.15 \mathrm{~ms}$

Vel Mag:

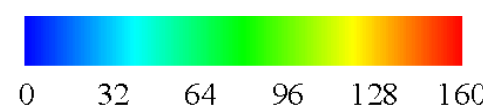

FIG. 7: Head-on PIV results for circular nozzle, $10 \mathrm{~mm}$ from nozzle exit, $P_{4} / P_{1}=12$. 


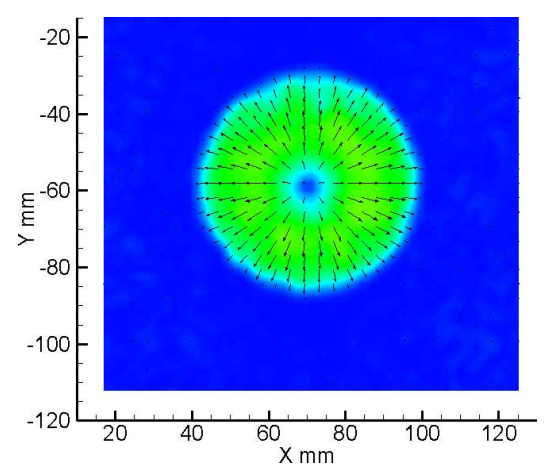

(a) $t=0.06 \mathrm{~ms}$

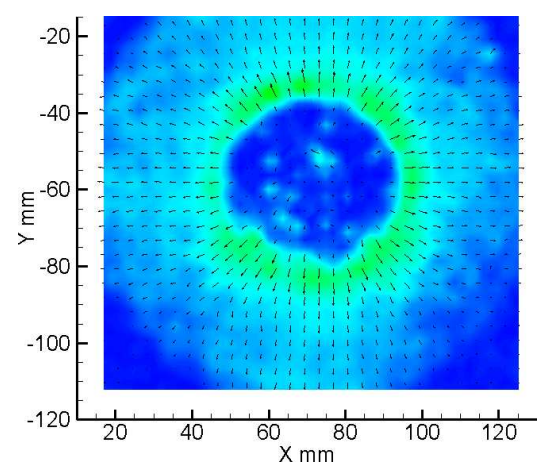

(d) $t=0.19 \mathrm{~ms}$

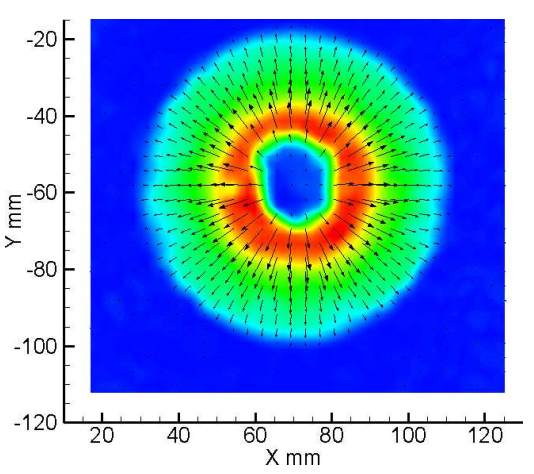

(b) $t=0.09 \mathrm{~ms}$

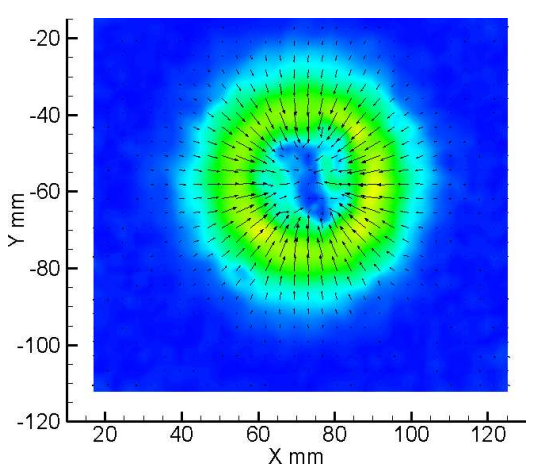

(e) $t=0.27 \mathrm{~ms}$

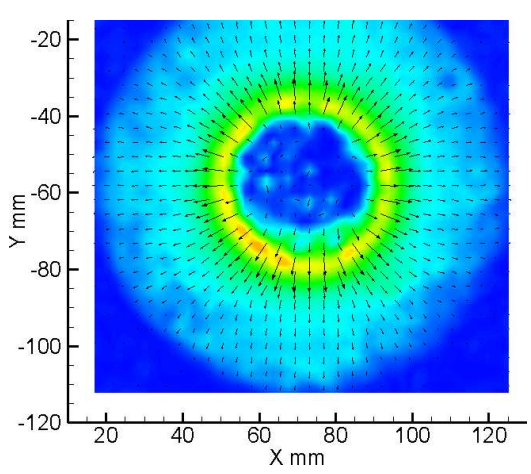

(c) $t=0.15 \mathrm{~ms}$

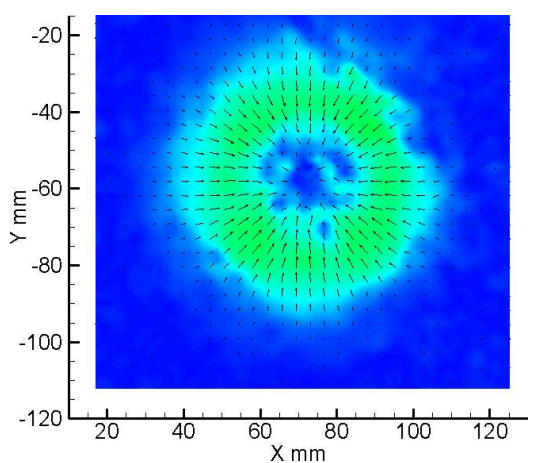

(f) $t=0.3 \mathrm{~ms}$

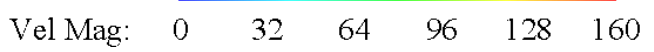

FIG. 8: Head-on PIV results for circular nozzle, $25 \mathrm{~mm}$ from nozzle exit, $P_{4} / P_{1}=12$.

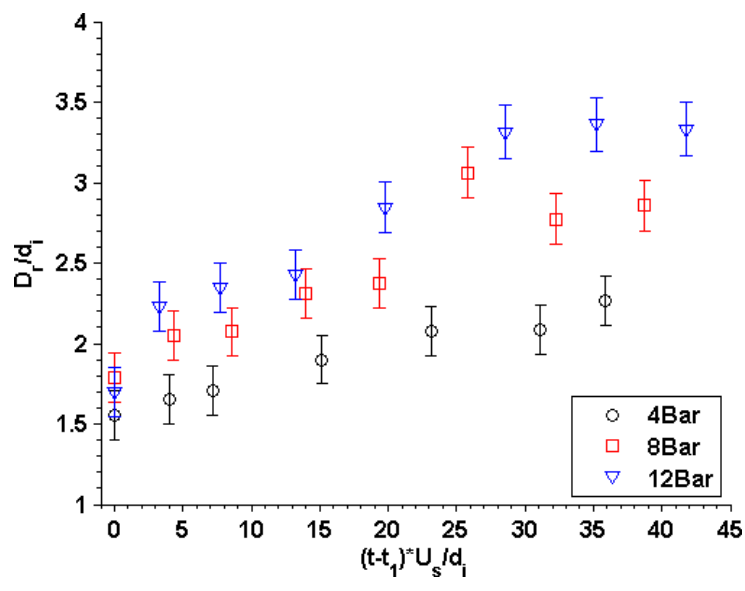

(a)

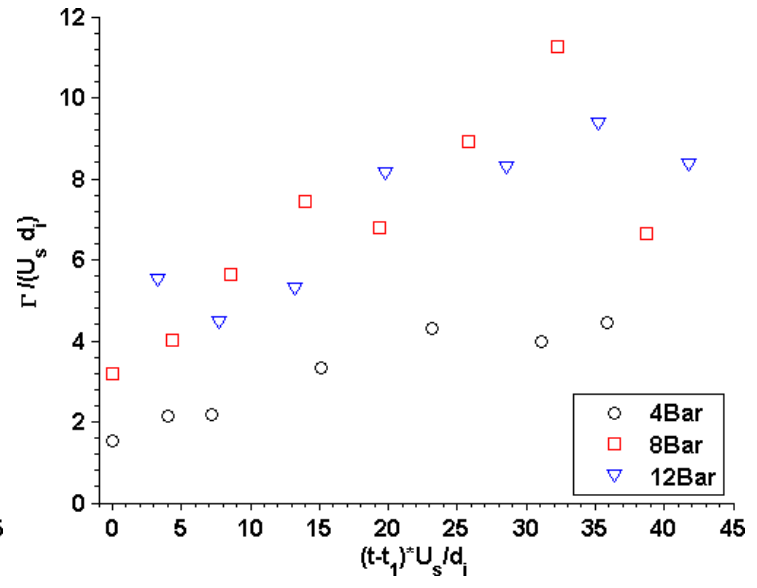

(b)

FIG. 9: Circular nozzle variation of: (a) vortex loop diameter, (b) vortex loop circulation. 


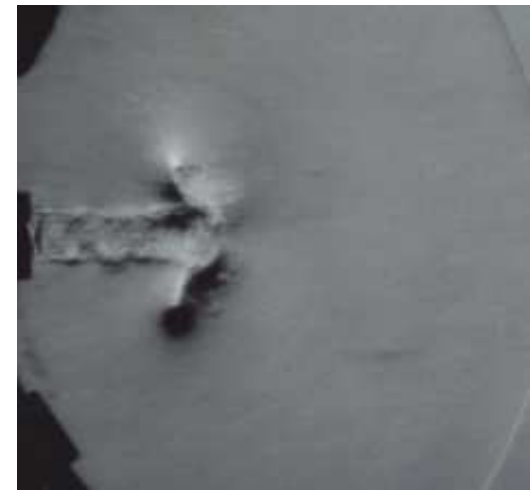

(a)

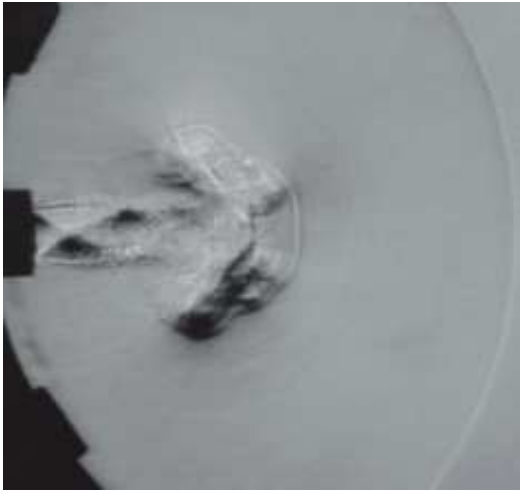

(b)

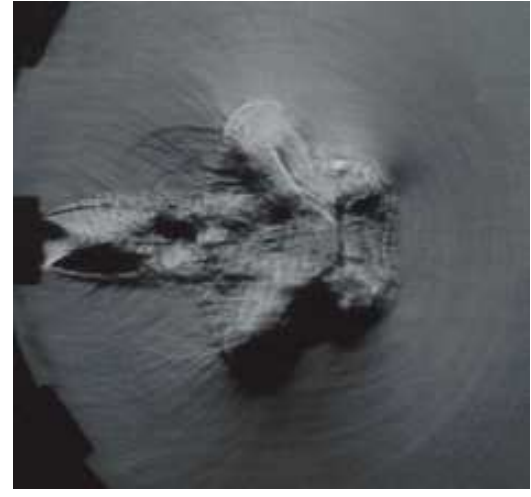

(c)

FIG. 10: Elliptic nozzle (0.4), side view, major axis horizontal, $P_{4} / P_{1}=$ (a) 4, (b) 8, (c) 12.

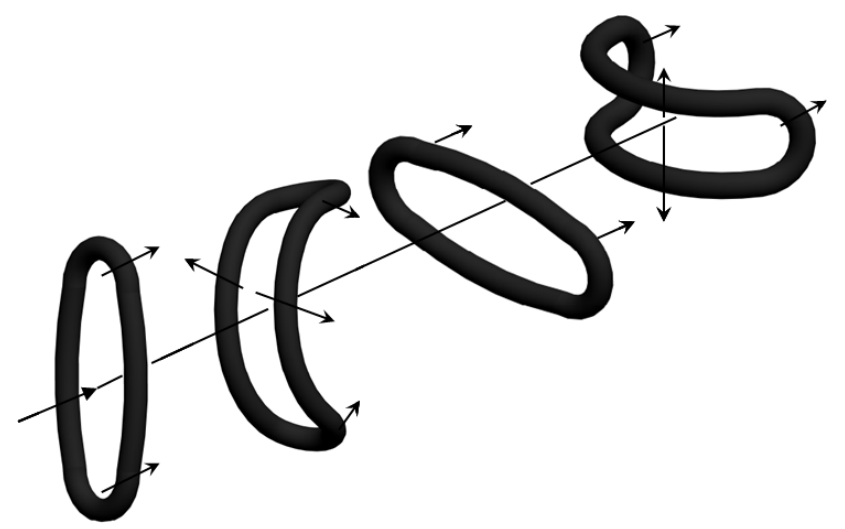

FIG. 11: Form and direction of motion of the individual parts of elliptic vortex loop.

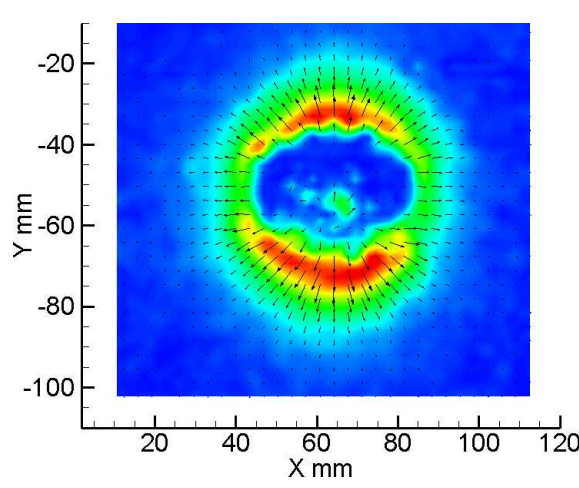

(a) $t=0.24 \mathrm{~ms}$

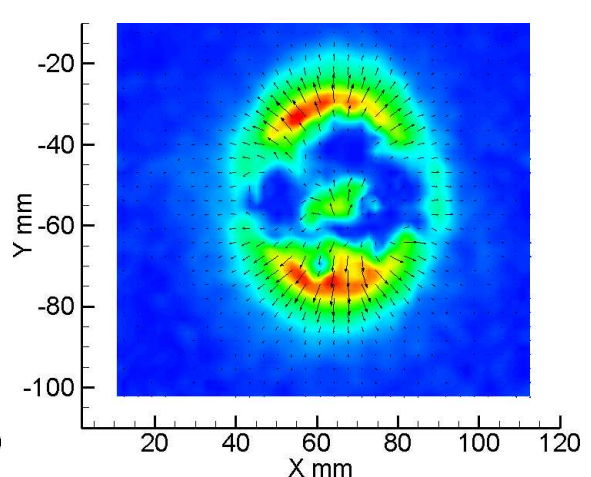

(b) $t=0.25 \mathrm{~ms}$

\section{$\begin{array}{lllllll}\text { Vel Mag: } & 0 & 24 & 48 & 72 & 96 & 120\end{array}$}

FIG. 12: Head-on PIV results for elliptic nozzle (0.4), $50 \mathrm{~mm}$ from nozzle exit, $P_{4} / P_{1}=4$. 


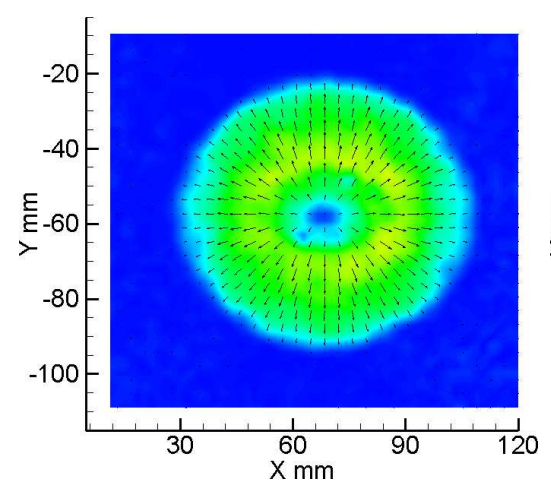

(a) $t=0.06 \mathrm{~ms}$

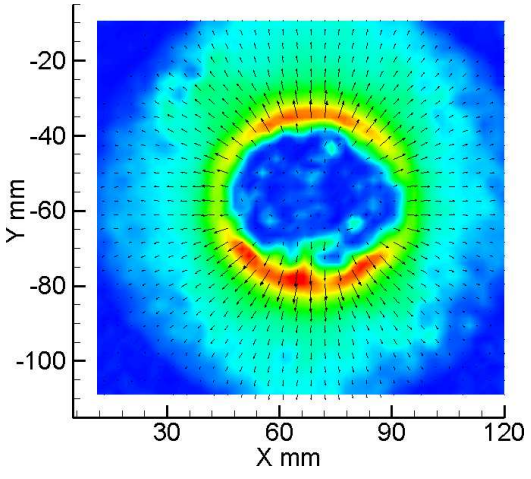

(b) $t=0.1 \mathrm{~ms}$

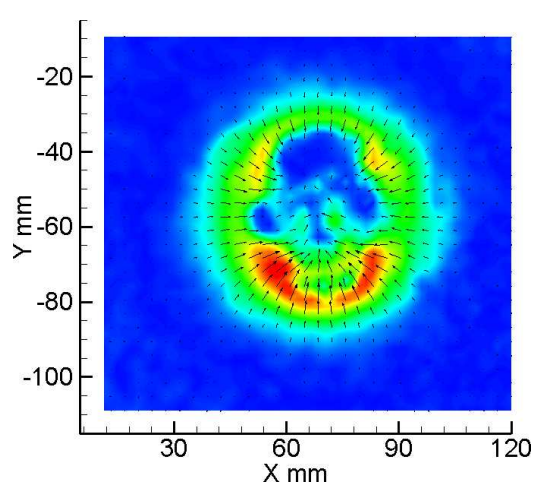

(c) $t=0.2 \mathrm{~ms}$

Vel Mag: $\quad \begin{array}{llllll}0 & 29 & 58 & 87 & 116 & 145\end{array}$

FIG. 13: Head-on PIV results for elliptic nozzle (0.4), $25 \mathrm{~mm}$ from nozzle exit, $P_{4} / P_{1}=12$.

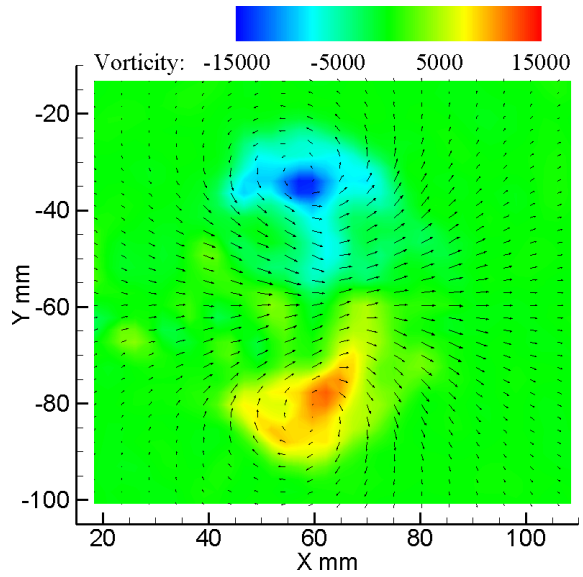

(a)

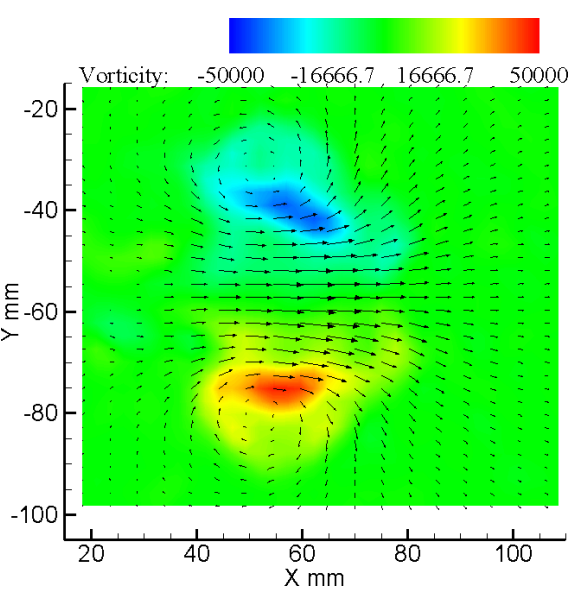

(b)

FIG. 14: PIV results for elliptic nozzle (0.4), side view, major axis horizontal, $P_{4} / P_{1}=(\mathrm{a}) 4$, (b) 12. 


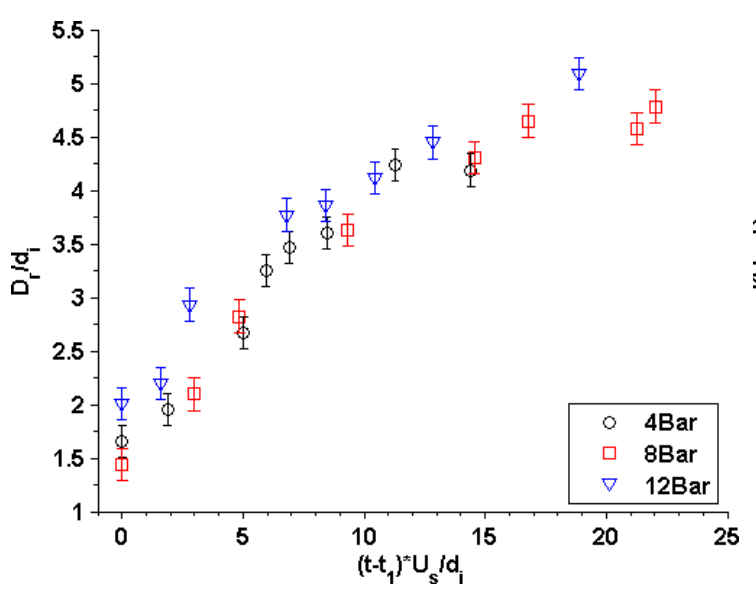

(a)

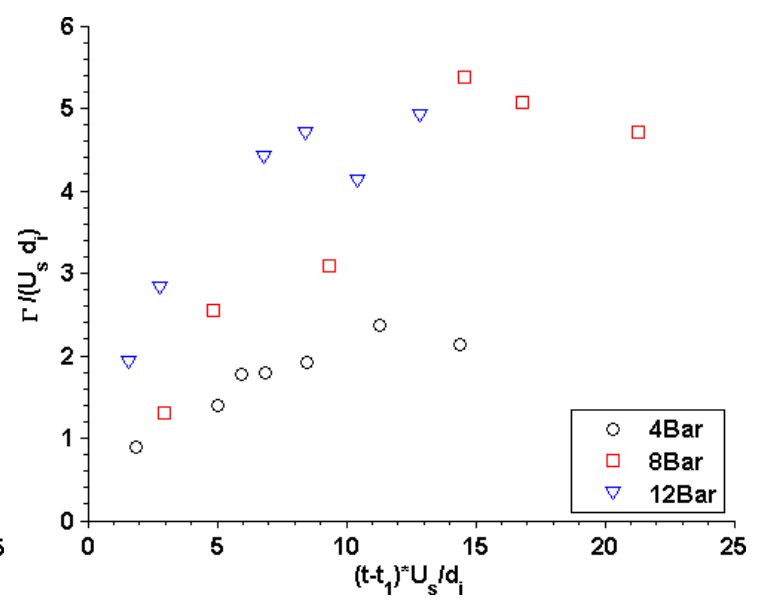

(b)

FIG. 15: Elliptic nozzle (0.4) variation of: (a) vortex loop diameter, (b) vortex loop circulation.

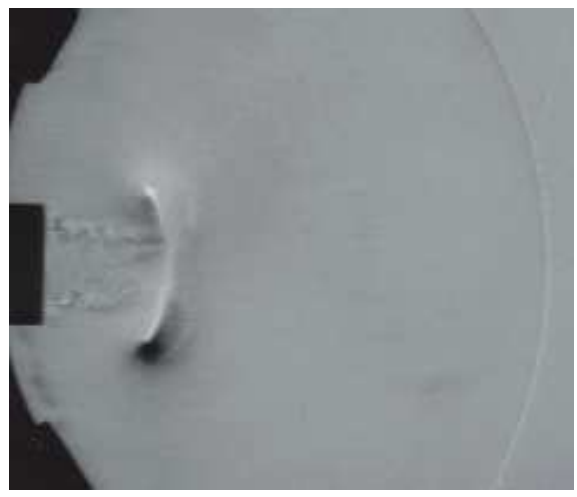

(a)

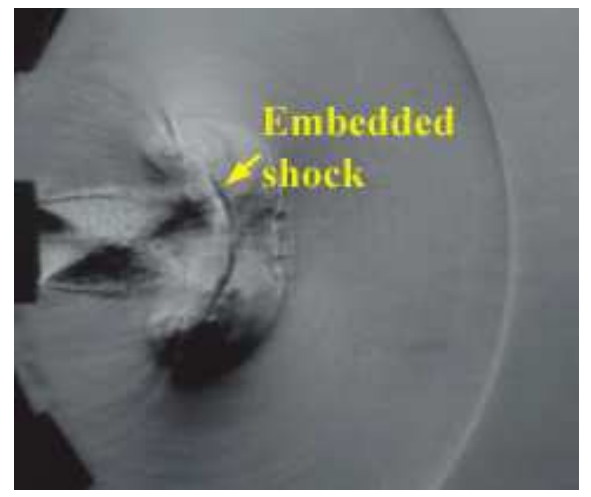

(b)

FIG. 16: Elliptic nozzle (0.6), side view, major axis horizontal, $P_{4} / P_{1}=$ (a) 4, (b) 12 . 


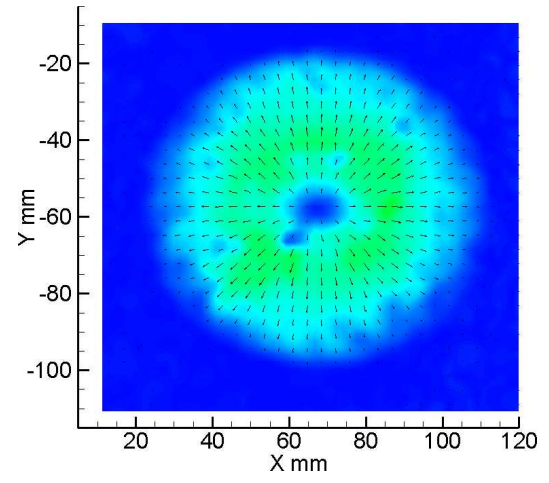

(a) $t=0.14 \mathrm{~ms}$

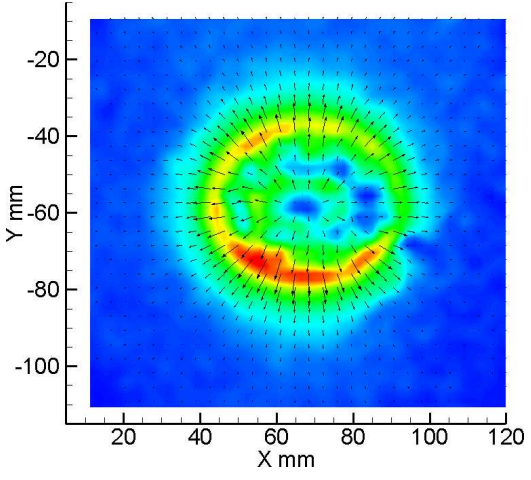

(b) $t=0.19 \mathrm{~ms}$

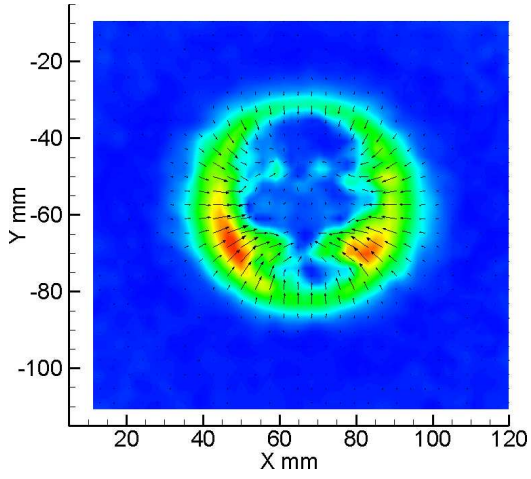

(c) $t=0.24 \mathrm{~ms}$

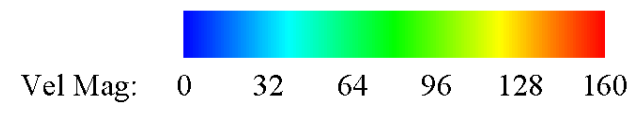

FIG. 17: Head-on PIV results for elliptic nozzle (0.6), $25 \mathrm{~mm}$ from nozzle exit, $P_{4} / P_{1}=8$. 


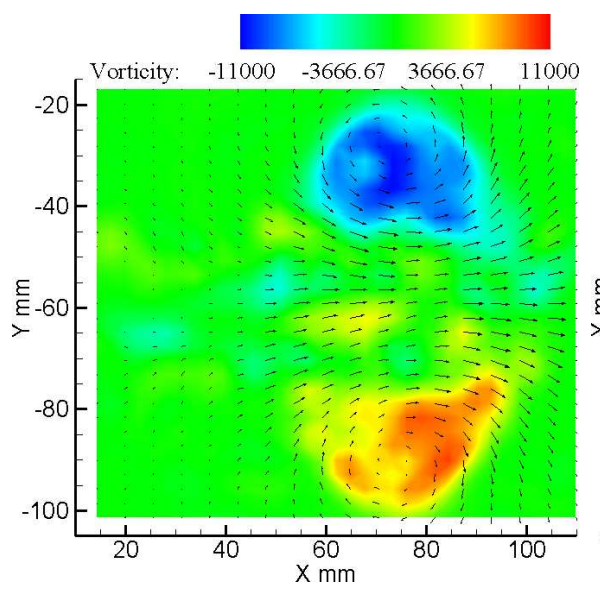

(a)

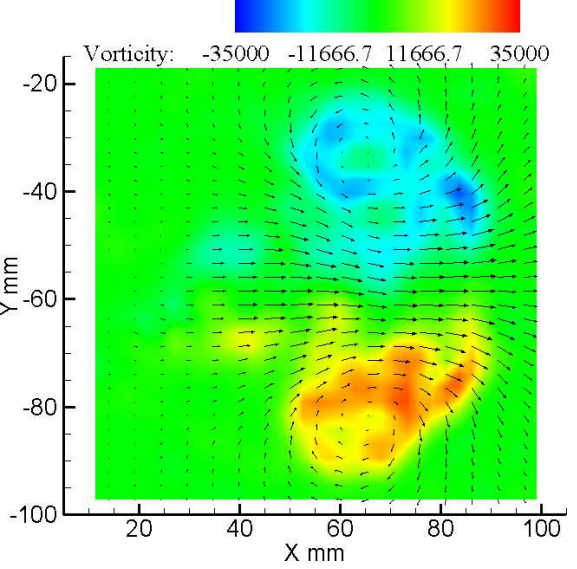

(b)

FIG. 18: PIV results for elliptic nozzle (0.6), side view, major axis horizontal, $P_{4} / P_{1}=(\mathrm{a}) 4$, (b) 8.

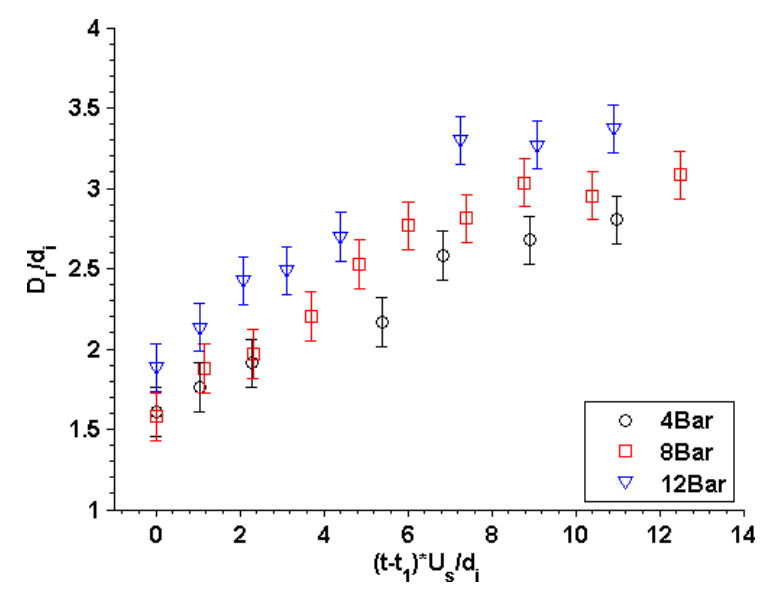

(a)

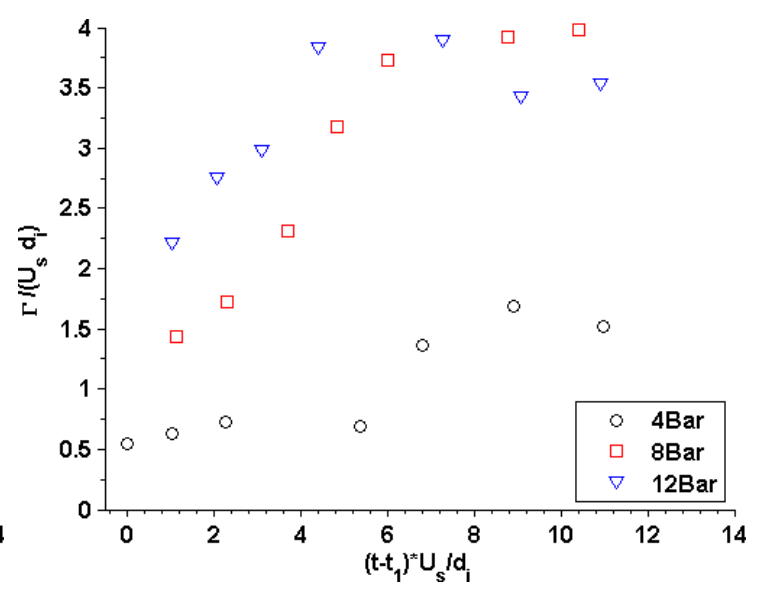

(b)

FIG. 19: Elliptic nozzle (0.6) variation of: (a) vortex loop diameter, (b) vortex loop circulation. 


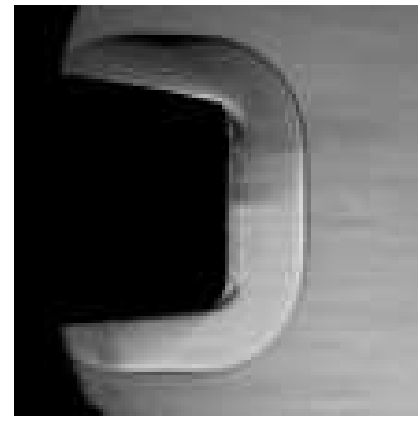

(a)

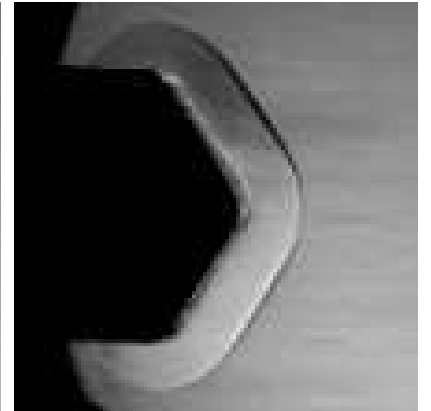

(b)

FIG. 20: Square nozzle, $P_{4} / P_{1}=12$, (a) oblique view, (b) oblique view with nozzle rotated (captured using the Shimadzu camera).

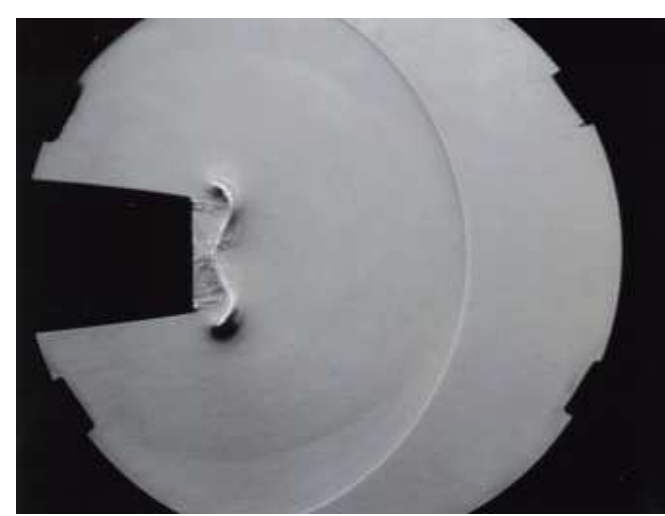

(a) $t=0.15 \mathrm{~ms}$

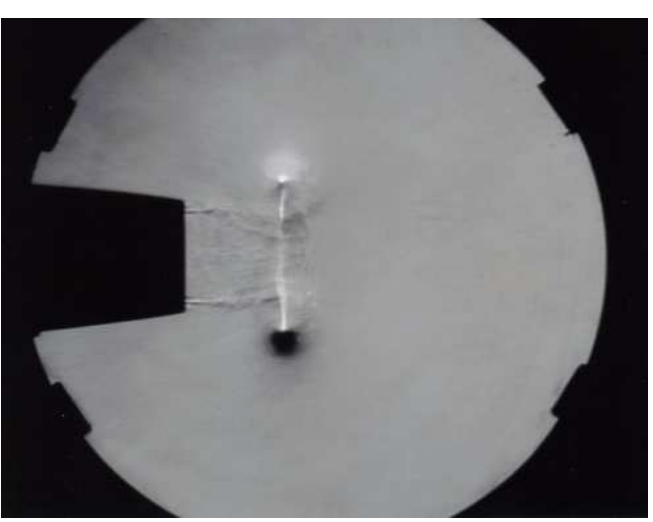

(b) $t=0.45 \mathrm{~ms}$

FIG. 21: Square nozzle, side view, $P_{4} / P_{1}=4$.

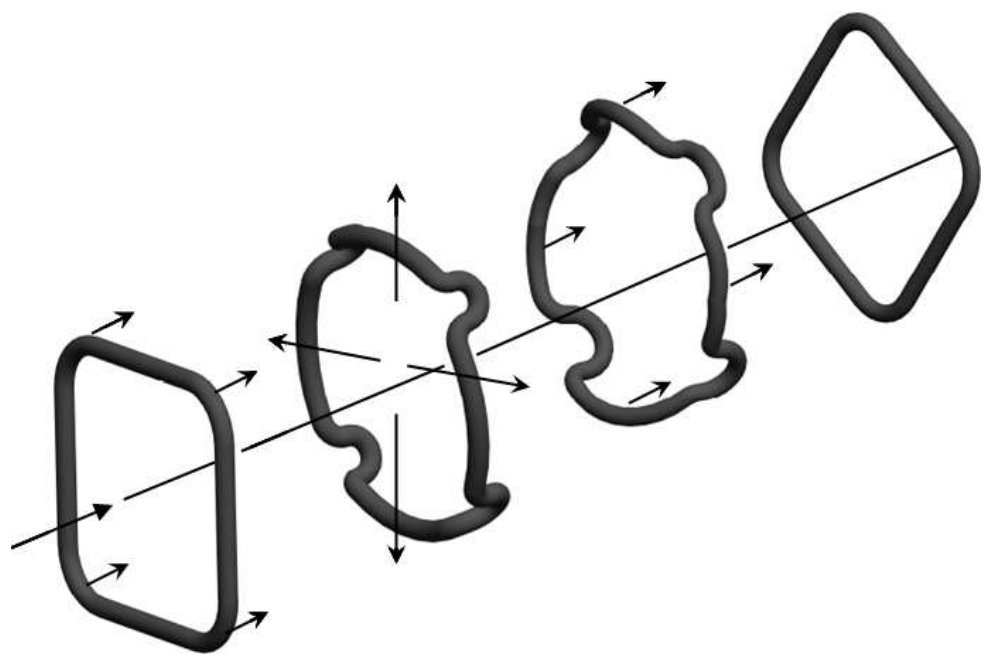

FIG. 22: Form and direction of motion of the individual parts of square vortex loop. 


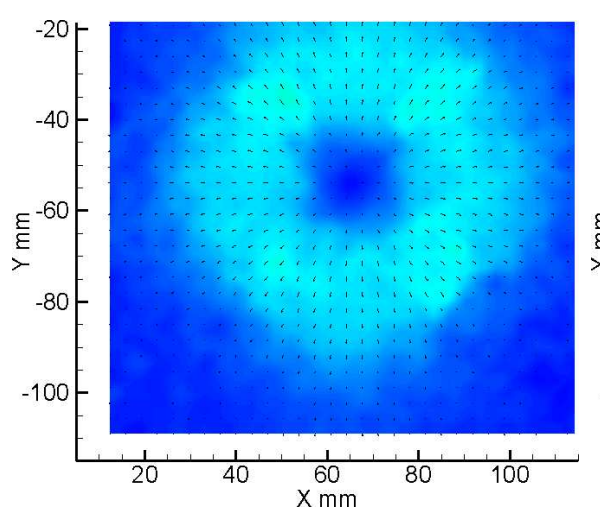

(a) $t=0.26 \mathrm{~ms}$

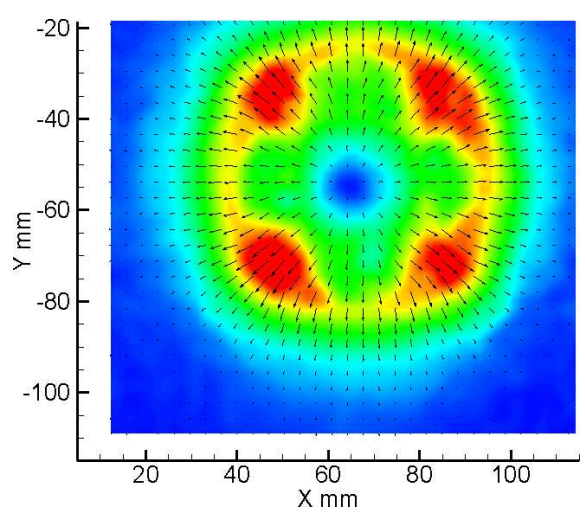

(c) $t=0.47 \mathrm{~ms}$

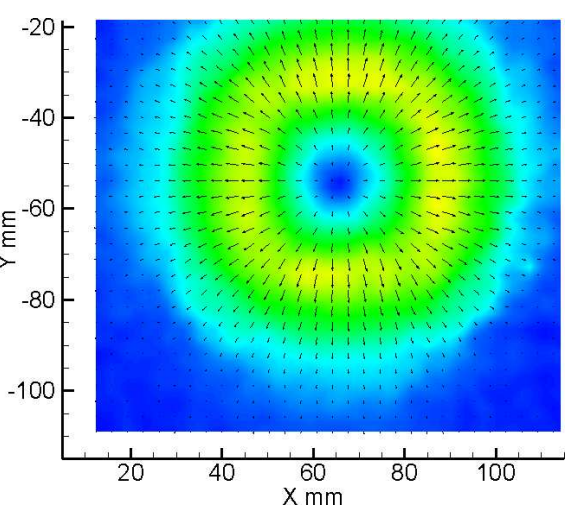

(b) $t=0.4 m s$

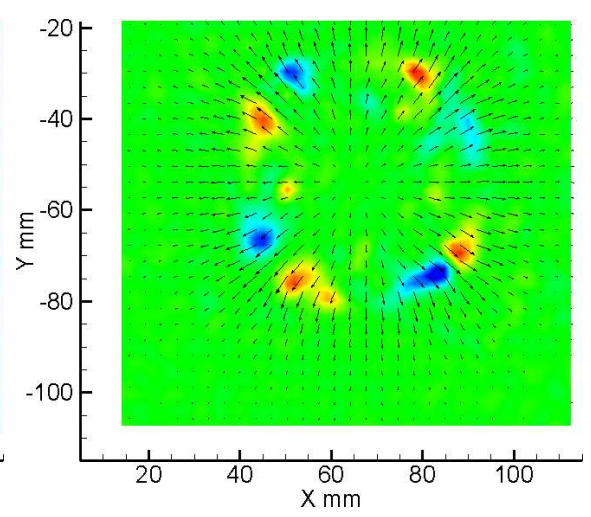

(d) $t=0.47 \mathrm{~ms}$

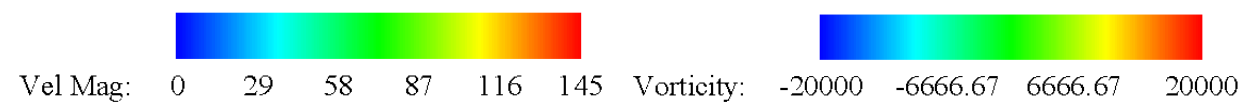

FIG. 23: Head-on PIV results for square nozzle, $50 \mathrm{~mm}$ from nozzle exit, $P_{4} / P_{1}=4$. 


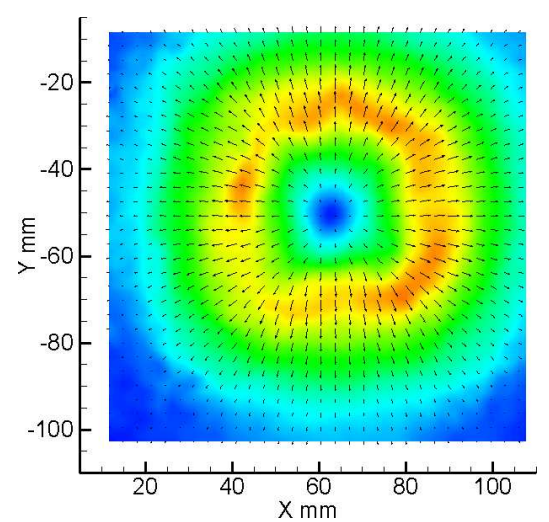

(a) $t=0.66 \mathrm{~ms}$

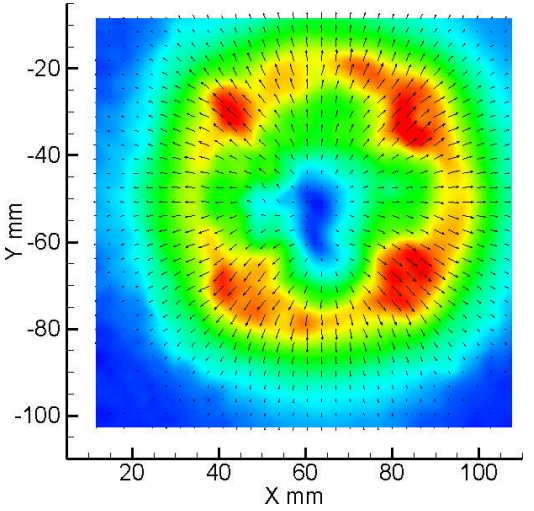

(b) $t=0.8 \mathrm{~ms}$

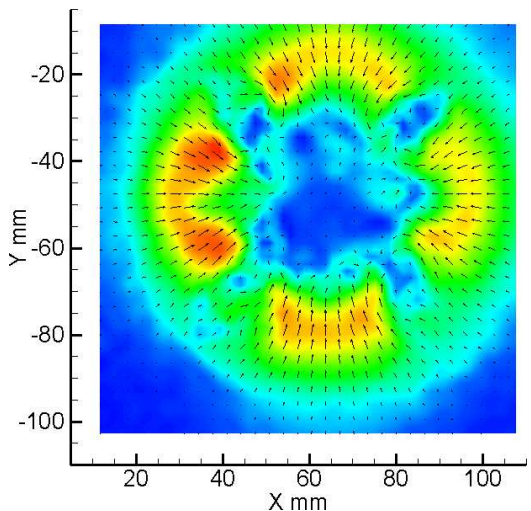

(c) $t=0.94 \mathrm{~ms}$

Vel Mag: $\quad \begin{array}{llllll}26 & 52 & 78 & 104 & 130\end{array}$

FIG. 24: Head-on PIV results for square nozzle, $80 \mathrm{~mm}$ from nozzle exit, $P_{4} / P_{1}=4$.

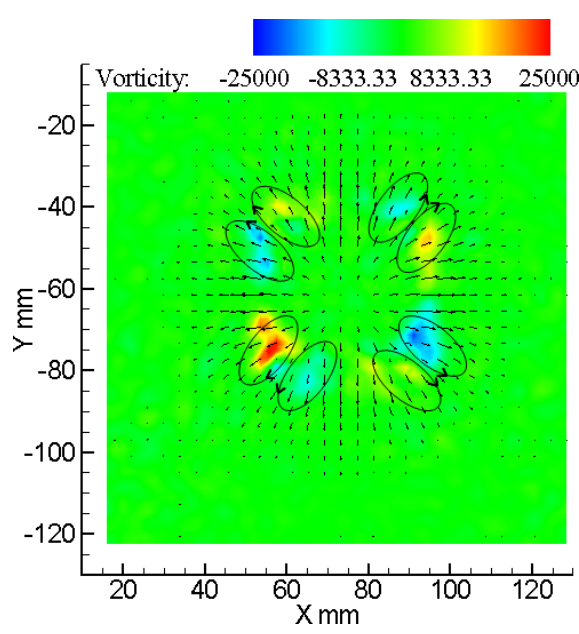

(a) $t=0.04 \mathrm{~ms}$

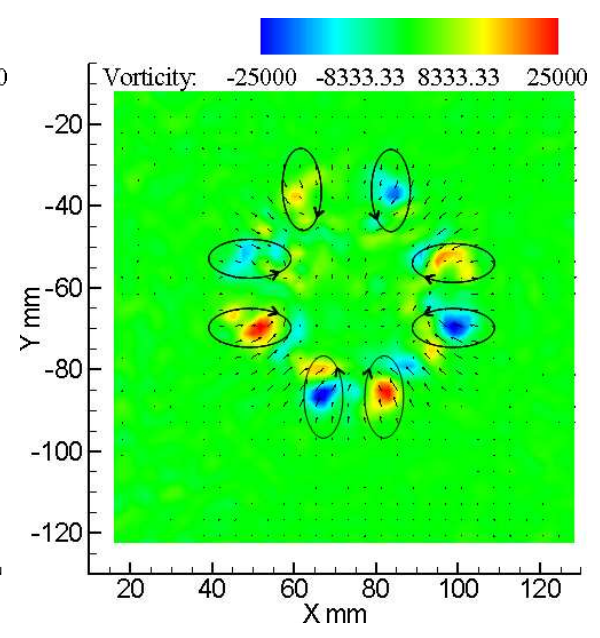

(b) $t=0.16 \mathrm{~ms}$

FIG. 25: Head-on PIV results for square nozzle, $10 \mathrm{~mm}$ from nozzle exit, $P_{4} / P_{1}=8$. 


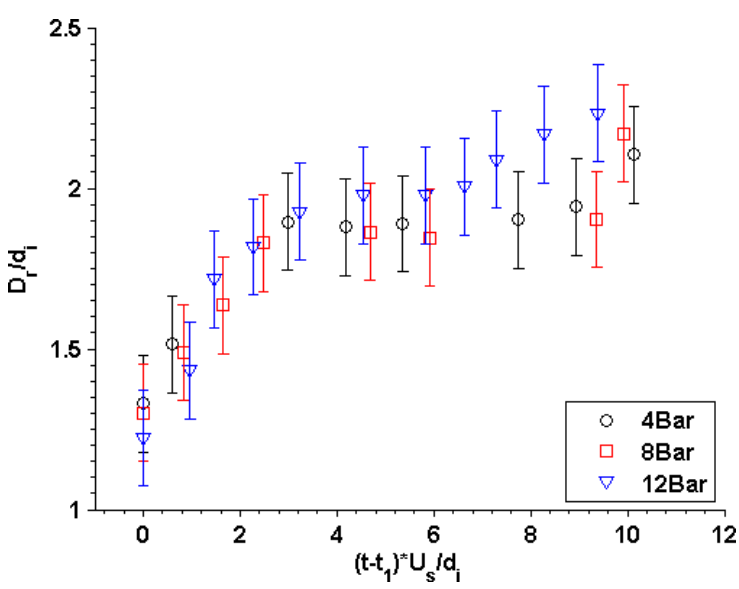

(a)

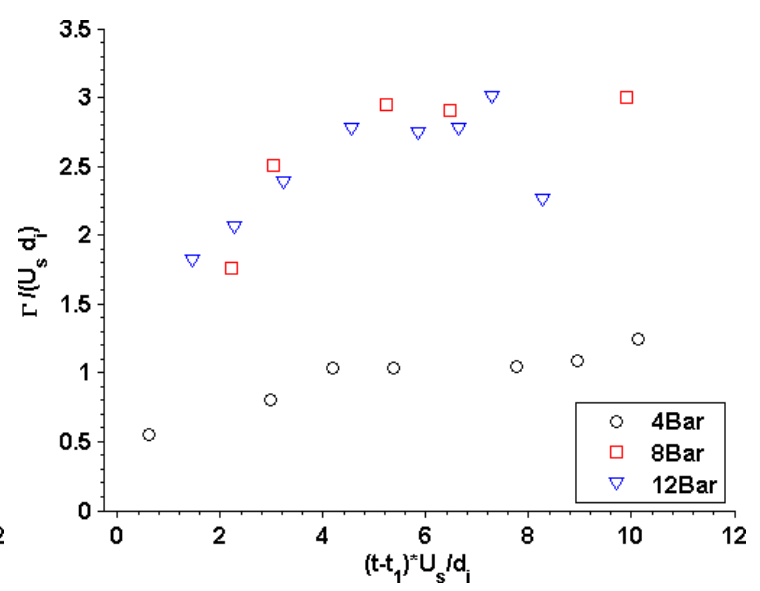

(b)

FIG. 26: Square nozzle variation of: (a) vortex loop diameter, (b) vortex loop circulation.

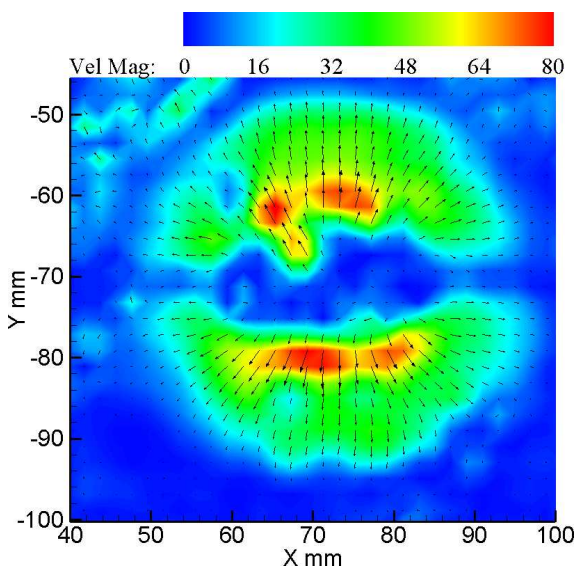

FIG. 27: Head-on PIV result for exotic nozzle (0.2), $10 \mathrm{~mm}$ from nozzle exit, $P_{4} / P_{1}=8$.

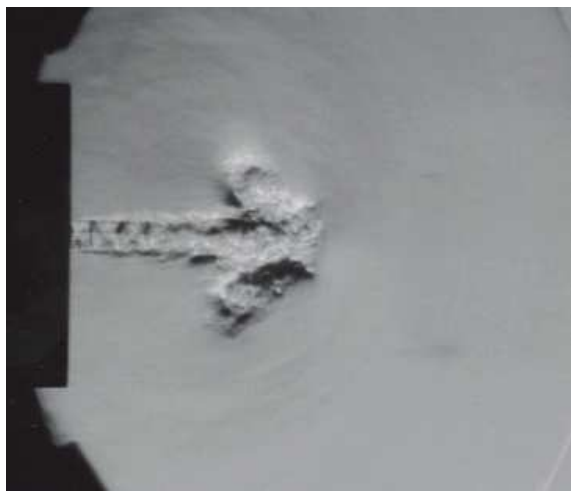

(a)

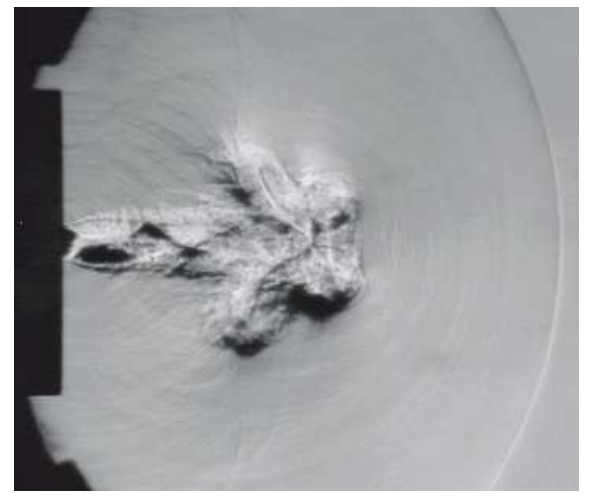

(b)

FIG. 28: Exotic nozzle (0.2), side view, major axis horizontal, $P_{4} / P_{1}=$ (a) 4, (b) 12. 


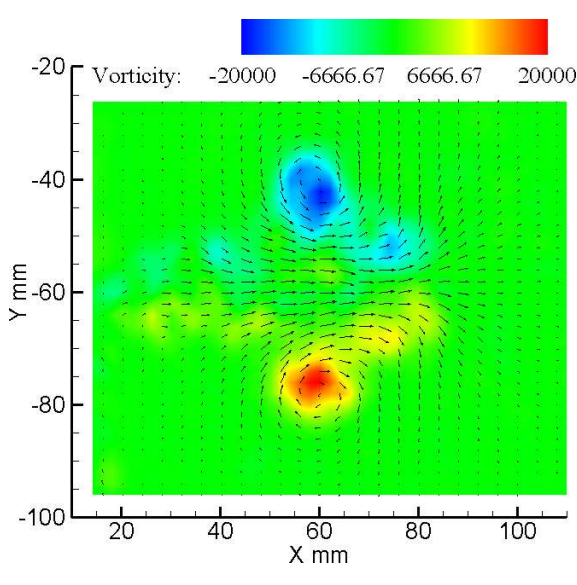

(a)

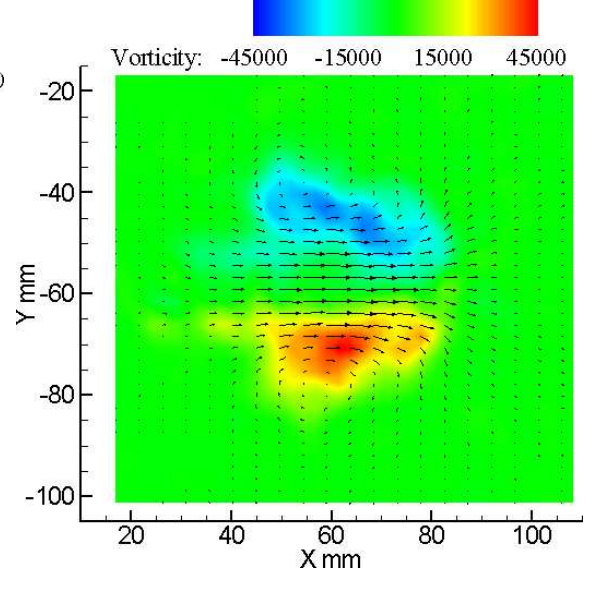

(b)

FIG. 29: PIV results for exotic nozzle (0.2), side view, major axis horizontal, $P_{4} / P_{1}=(\mathrm{a}) 4$, (b) 12.

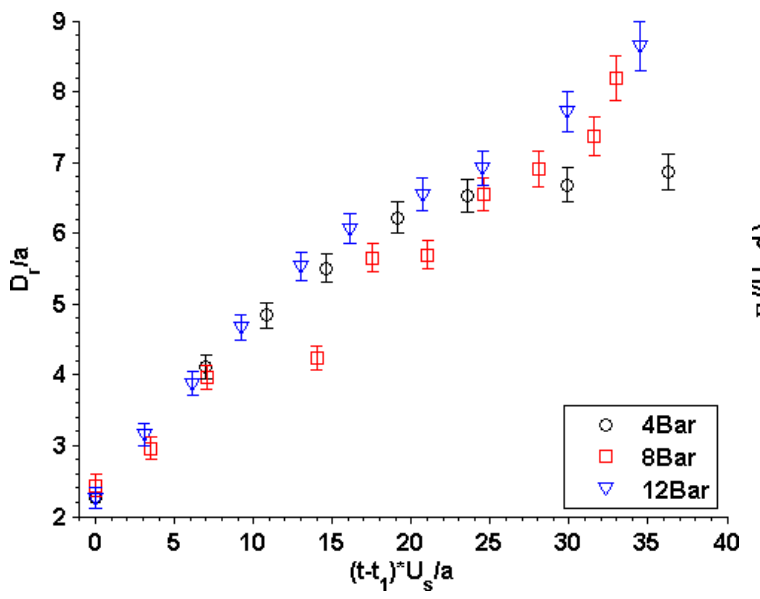

(a)

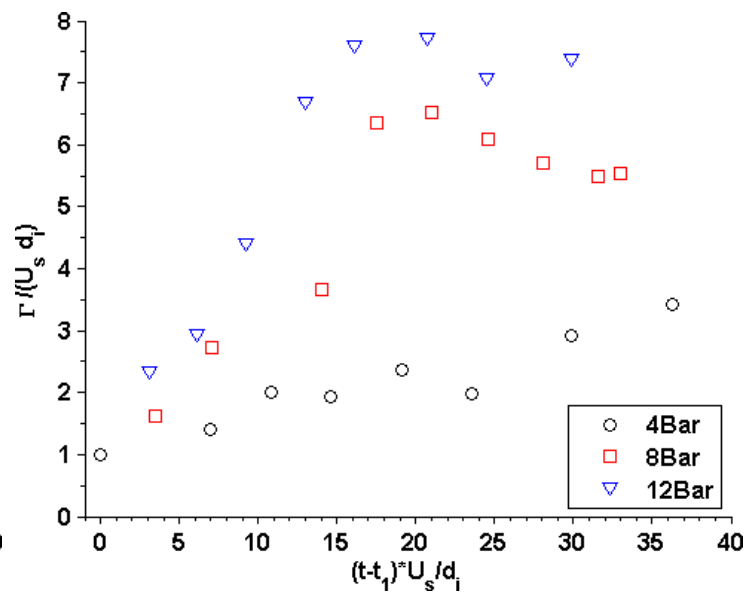

(b)

FIG. 30: Exotic nozzle (0.2) variation of: (a) vortex loop diameter, (b) vortex loop circulation. 


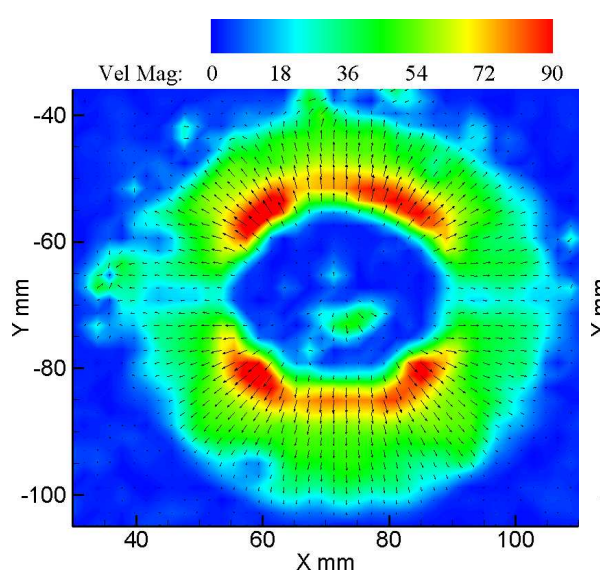

(a) $t=0.08 \mathrm{~ms}$

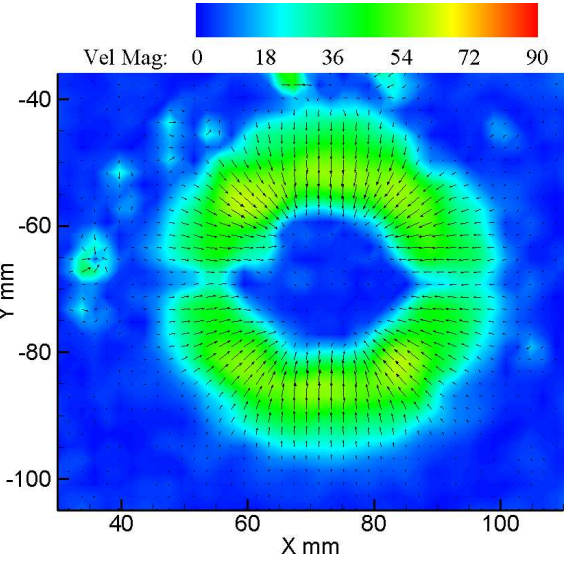

(b) $t=0.15 \mathrm{~ms}$

FIG. 31: Head-on PIV results for exotic nozzle (0.5), $10 \mathrm{~mm}$ from nozzle exit, $P_{4} / P_{1}=8$.

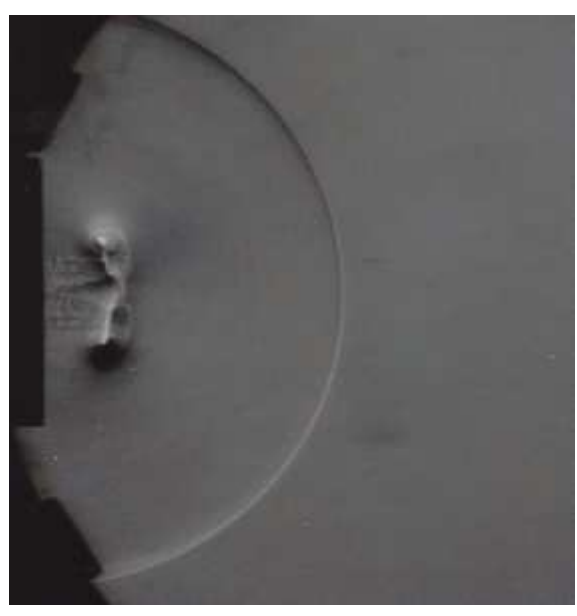

(a)

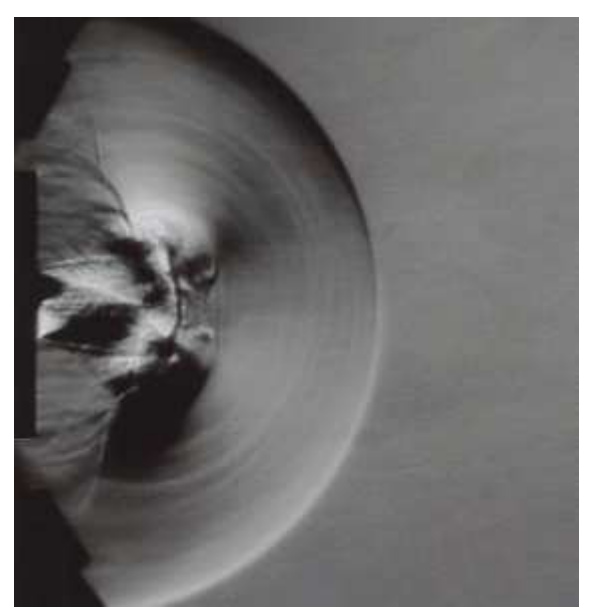

(b)

FIG. 32: Exotic nozzle (0.5), side view, major axis horizontal, $P_{4} / P_{1}=$ (a) 4, (b) 12. 


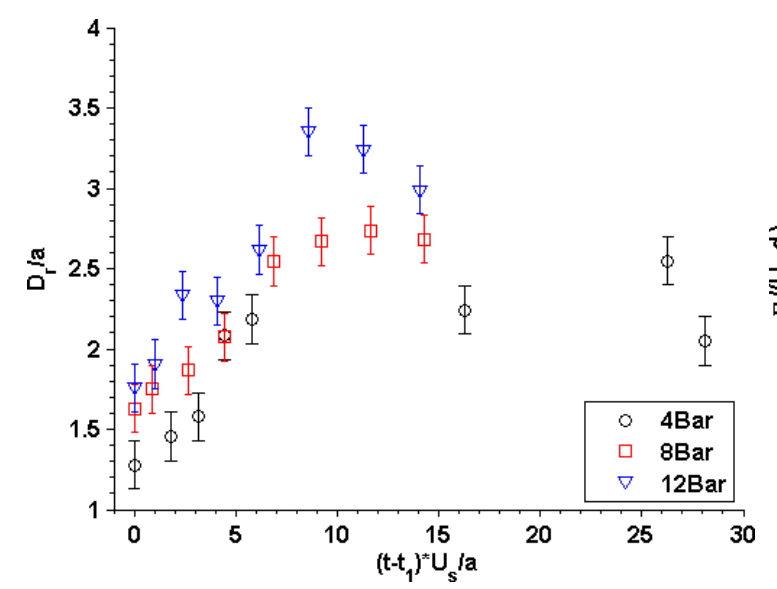

(a)

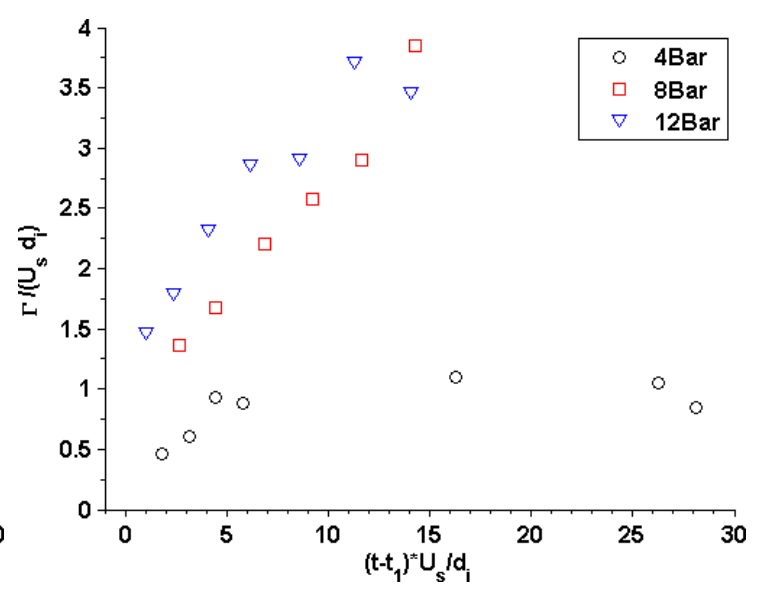

(b)

FIG. 33: Exotic nozzle (0.5) variation of: (a) vortex loop diameter, (b) vortex loop circulation.

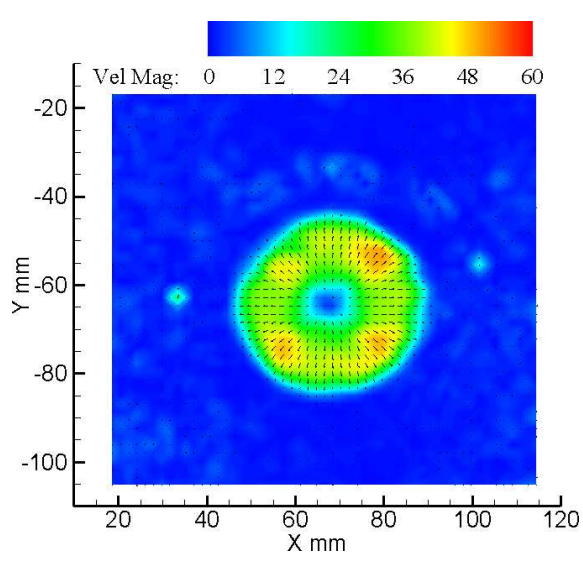

(a) $t=0.08 \mathrm{~ms}$

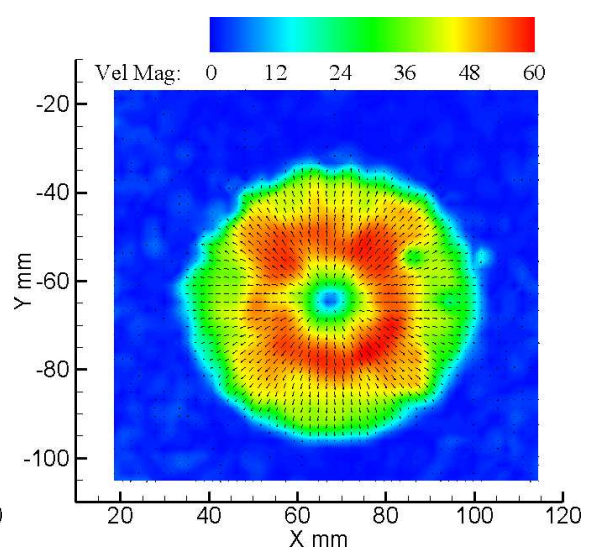

(b) $t=0.1 \mathrm{~ms}$

FIG. 34: Head-on PIV results for exotic nozzle (0.5), $25 \mathrm{~mm}$ from nozzle exit, $P_{4} / P_{1}=8$. 


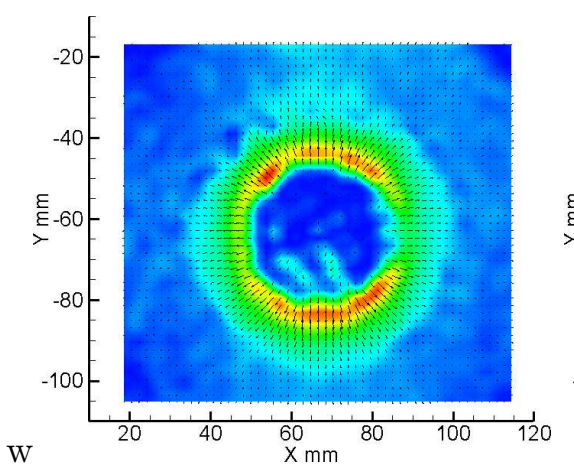

(a) $t=0.19 \mathrm{~ms}$

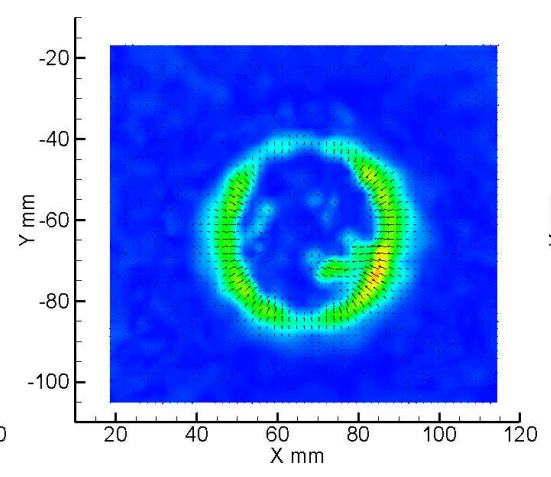

(b) $t=0.22 \mathrm{~ms}$

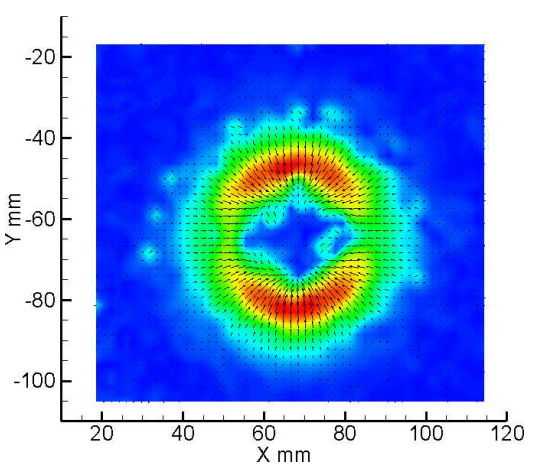

(c) $t=0.26 \mathrm{~ms}$

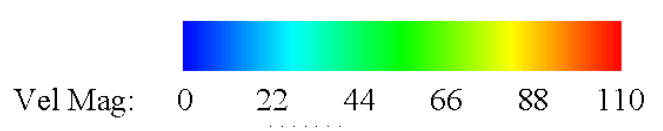

FIG. 35: Head-on PIV results for exotic nozzle (0.5), $25 \mathrm{~mm}$ from nozzle exit, $P_{4} / P_{1}=8$.

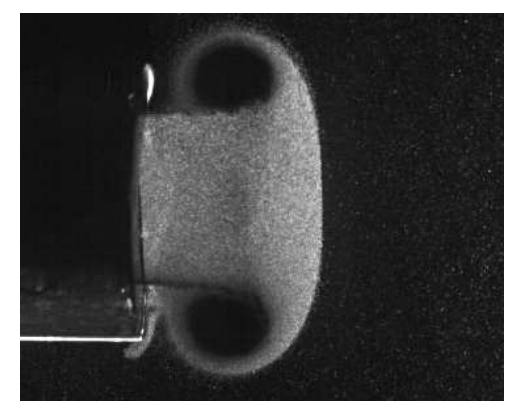

(a)

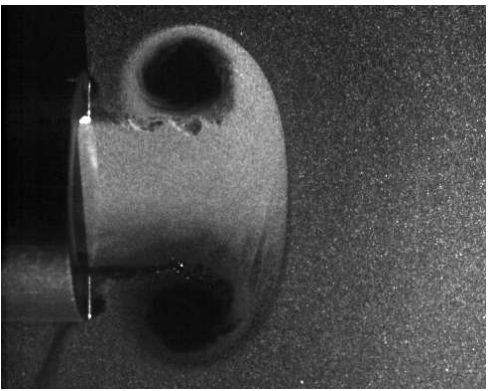

(b)

FIG. 36: Laser induced fluorescence: (a) circular vortex loop, (b) elliptic vortex loop (0.4). 